\title{
Optimizing detector geometry for trace element mapping by X-ray fluorescence
}

\author{
Yue Sun ${ }^{\mathrm{a}}$, Sophie-Charlotte Gleber ${ }^{\mathrm{b}}$, Chris Jacobsen ${ }^{\mathrm{b}, \mathrm{d}, \mathrm{e}}$, Janos Kirz ${ }^{\mathrm{c}}$, Stefan Vogt ${ }^{\mathrm{b}, *}$ \\ ${ }^{a}$ Graduate Program in Applied Physics, Northwestern University, Evanston, IL 60208, USA \\ ${ }^{b}$ Argonne National Laboratory, Argonne, IL 60439, USA \\ ${ }^{c}$ Lawrence Berkeley National Laboratory, Berkeley, CA 94720, USA \\ ${ }^{d}$ Department of Physics and Astronomy, Northwestern University, Evanston, IL 60208, USA \\ ${ }^{e}$ Chemistry of Life Processes Institute, Northwestern University, Evanston, IL 60208, USA
}

\begin{abstract}
Trace metals play critical roles in a variety of systems, ranging from cells to photovoltaics. X-Ray Fluorescence (XRF) microscopy using X-ray excitation provides one of the highest sensitivities available for imaging the distribution of trace metals at sub-100 nm resolution. With the growing availability and increasing performance of synchrotron light source based instruments and X-ray nanofocusing optics, and with improvements in energy-dispersive XRF detectors, what are the factors that limit trace element detectability? To address this question, we describe an analytical model for the total signal incident on XRF detectors with various geometries, including the spectral response of energy dispersive detectors. This model agrees well with experimentally recorded X-ray fluorescence spectra, and involves much shorter calculation times than with Monte Carlo simulations. With such a model, one can estimate the signal when a trace element is illuminated with an X-ray beam, and when just the surrounding non-fluorescent material is illuminated. From this signal difference, a contrast parameter can be calculated and this can in turn be used to calculate the signal-to-noise ratio $(\mathrm{S} / \mathrm{N})$ for detecting a certain elemental concentration. We apply this model to the detection of trace amounts of zinc in biological materials, and to the detection of small quantities of arsenic in semiconductors. We conclude that increased detector collection solid angle is (nearly) always advantageous even when considering the scattered signal. However, given the choice between a smaller detector at $90^{\circ}$ to the beam versus a larger detector at $180^{\circ}$ (in a backscatter-like geometry), the $90^{\circ}$ detector is better for trace element detection in thick samples, while the larger detector in $180^{\circ}$ geometry is better suited to trace element detection in thin samples.
\end{abstract}

Keywords: trace element detection, X-ray fluorescence, X-ray fluorescence microscopy, detector geometry, signal-to-noise ratio

\footnotetext{
${ }^{*}$ Corresponding author

Email addresses: yuesun2017@u.northwestern.edu (Yue Sun), gleber@aps.anl.gov (Sophie-Charlotte Gleber), cjacobsen@anl.gov (Chris Jacobsen), jkirz@lbl.gov (Janos Kirz), vogt@aps.anl.gov (Stefan Vogt) 


\section{Introduction}

Trace elements play an important role in many aspects of our world. Cells and organisms require trace metals for their normal function; for example, roughly one third of all proteins require a metal atom or cofactor to function properly. However, excess exposure to, deficiency of, or disregulation of trace metals are 5 all implicated in various diseases; several examples have been investigated by X-ray fluorescence microscopy $[1,2,3,4,5]$. Trace elements in the environment can affect the functioning of microbes, animals, and plants $[6,7,8]$. In manufactured materials such as polycrystalline photovoltaics, trace metals can significantly degrade device performance $[9,10]$; in the semiconductor devices that pervade our lives, trace elements such as arsenic are essential for device operation. Trace elements even control the color and perceived value of gemstones.

There are a wide variety of techniques for measuring trace elemental distribution, including mass spectrometry (for example with micrometer-scale matrix-assisted laser desorption, or nanoscale focused ion beam desorption) and atomic emission spectroscopy. The measurement of characteristic X-ray emission lines (Xray fluorescence, or XRF) is among the most sensitive, and moreover one can stimulate XRF in a relatively non-destructive manner. For XRF measurements, one must remove core shell electrons so that fluorescence is generated as electrons from less strongly bound states drop down to fill the vacancy. Inner-shell ionization is usually accomplished by using proton, electron or X-ray probes. With Proton-Induced X-ray Emission (PIXE) [11, 12], one has the advantage that the high mass of protons leads to relatively low continuum background emission, so X-ray fluorescence lines are readily detectable above a very low background signal; the downside is that the proton mass also leads to considerably more specimen damage than one would have using excitation beams with lower momentum transfer. Electron Probe Micro-Analysis (EPMA) [13, 14] are based on scanning electron microscopes equipped with energy or wavelength dispersive fluorescence detectors; they impart less damage to the specimen, but weaker fluorescence lines can become obscured by the continuum background signal, spatial resolution can be affected by electron beam sidescattering, and typically thin sections are required. By using focused X-ray beams to remove core level electrons via X-ray absorption, one has the advantage that there is a much lower background signal leading to improved trace element sensitivity at reduced radiation dose $[15,16,17,18]$, as well as no beam blurring due to beam scattering. We therefore concentrate in this paper on a more detailed examination of trace element analysis in X-ray microprobes.

While the general advantageous properties of XRF analysis in X-ray microprobes are well-known [19], a detailed understanding involves a sequence of interactions as will be shown below. The best approach is to fully account for all possible interactions in Monte Carlo simulations, and considerable success has been obtained with excellent agreement with experiments. These include fundamental momentum based Monte Carlo simulations intended for general scattering phenomena, like GEANT4 [20] and its derivatives [21, 
22], and more specific interaction cross-section based simulations for energy-dispersive XRF (ED-XRF) spectrometers $[23,24,25,26,27,28]$. However, while Monte Carlo simulations can deliver a very detailed view of what can be expected from one or a few experimental configurations, we consider here an analytical approach which allows for more rapid exploration of a greater range of experimental parameters. Since there have been new developments in fast data collection with scanned beams [29], and fluorescence detectors with new geometries and data acquisition strategies [30, 31, 32], this faster analytical approach provides a tool to explore a wider range of experimental configurations so as to optimize trace element analysis for a different specimen types and using different types of detectors. We compare our approach against Monte Carlo simulations and also against experimental data, and find a very satisfactory agreement. We then apply this approach to consider trace element detection in two example specimens shown in Figure 1:

1. "Bio" specimen: this sample consists of a $20 \mathrm{~nm}$ thick layer with the stochiometric elemental distribution of a typical protein $\left(\mathrm{C}_{30} \mathrm{H}_{50} \mathrm{O}_{10} \mathrm{~N}_{9} \mathrm{~S}\right)$ containing $0.01 \% \mathrm{Zn}$ by weight, sandwiched between two water layers of varying thickness so as to comprise a specified total sample thickness. This sample approximates a small organelle within a cell, where Zn can play roles in fertilization and signaling (see for example [33]).

2. "Chip" specimen: this sample consists of a $0.5 \mathrm{~nm}$ thick layer of As on top of a Si crystal with specified thickness. Arsenic is a common dopant used in semiconductor integrated circuits, or silicon "chips."

We will refer to these "bio" and "chip" specimens below.

(a)

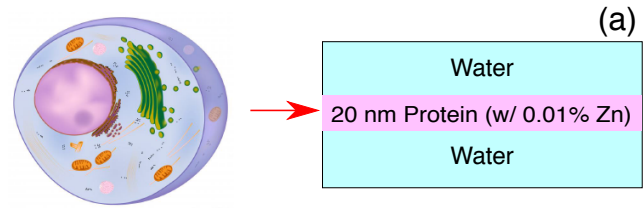

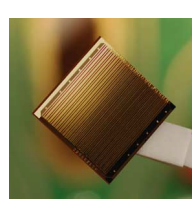

(b)

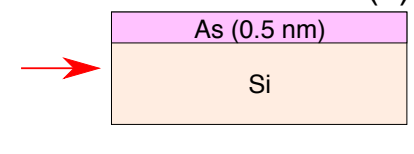

Figure 1: Two models used as test samples. (a) The "Bio" sample consists of a thin (20 nm) protein layer with 0.01 wt\% of Zn sandwiched between two layers of water to comprise a total sample thickness. (b) The "Chip" modeled semiconductor sample consists of a $0.5 \mathrm{~nm}$ thick layer of As on top of a Si layer with specified thickness.

\section{X-ray fluorescence and signal estimates}

Because synchrotron light sources usually make use of magnetic beam deflections in the horizontal plane, most beamlines (X-ray beam delivery systems) produce X-ray beams that are linearly polarized in the horizontal direction. With these polarized beams, there is a minimum in the elastic and inelastic (or Compton) scattering cross section at $90^{\circ}$ from the incident beam direction, so that detectors mounted in the $90^{\circ}$ configuration (Figure 2) see a minimum of scattered photons as a background signal. It is for this 
reason that most synchrotron XRF microprobes are operated in the $90^{\circ}$ geometry, in spite of associated inconveniences as discussed in Section 2.2. With detectors in the $90^{\circ}$ geometry, increasing the detector's solid angle coverage will increase the strength of the detected fluorescence signals, but it will also increase the scattering background signal. How does this scale with fluorescence detector aperture? Is there an optimum solid angle of acceptance? In addition, newer detector options have emerged: one is the Maia detector $[31,34,32]$, which is typically deployed in the $180^{\circ}$ geometry, so that it is equipped with a hole in its center to allow the X-ray nanofocused beam to reach the specimen. What are the advantages and drawbacks of this geometry? These are some of the questions we wish to address.

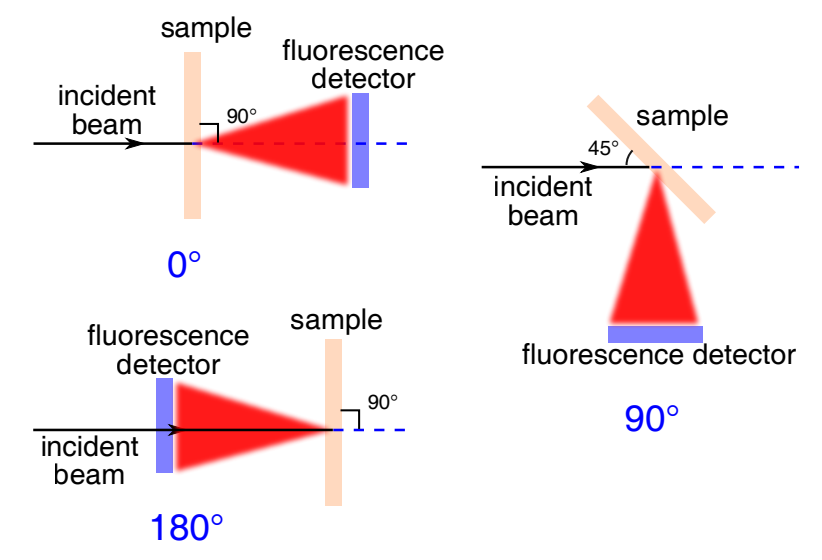

Figure 2: Three common detector geometries used for X-ray fluorescence detection at synchrotron light sources. These geometries have the detectors positioned at $0^{\circ}, 90^{\circ}$, or $180^{\circ}$ to the incident beam. Elastic and inelastic scattering of the $\mathrm{X}$-ray beam is minimized at $90^{\circ}$ due to the polarization of synchrotron radiation, while the $180^{\circ}$ or "backscatter" geometry has advantages for easier scanning of large specimens.

We consider below several factors that lead to the final detected signal and background in XRF analysis, in the case of incident radiation with perfect plane polarization:

- Scattering of the incident X-ray beam. While elastic or Rayleigh scattering is completely polarization dependent and minimized to zero at $90^{\circ}$ to the polarized incident X-ray beam, inelastic or Compton X-ray scattering has a different distribution as described by Klein and Nishina [35, 36] combined with the incoherent scattering function [37], which contains both polarization dependent and independent components leading to a non-zero minimum at $90^{\circ}$ (Figure 3 ).

- Absorption by the atoms to be detected, and the emission of X-ray fluorescent photons versus Auger electrons $[38,39]$ as well as their possible reabsorption.

- The response of energy-dispersive X-ray detectors, including energy spread and incomplete charge collection. 
With an estimate of detected signal and background in hand, we can estimate the signal-to-noise ratio (S/N) and then estimate the number of incident photons that are required for trace element detection.

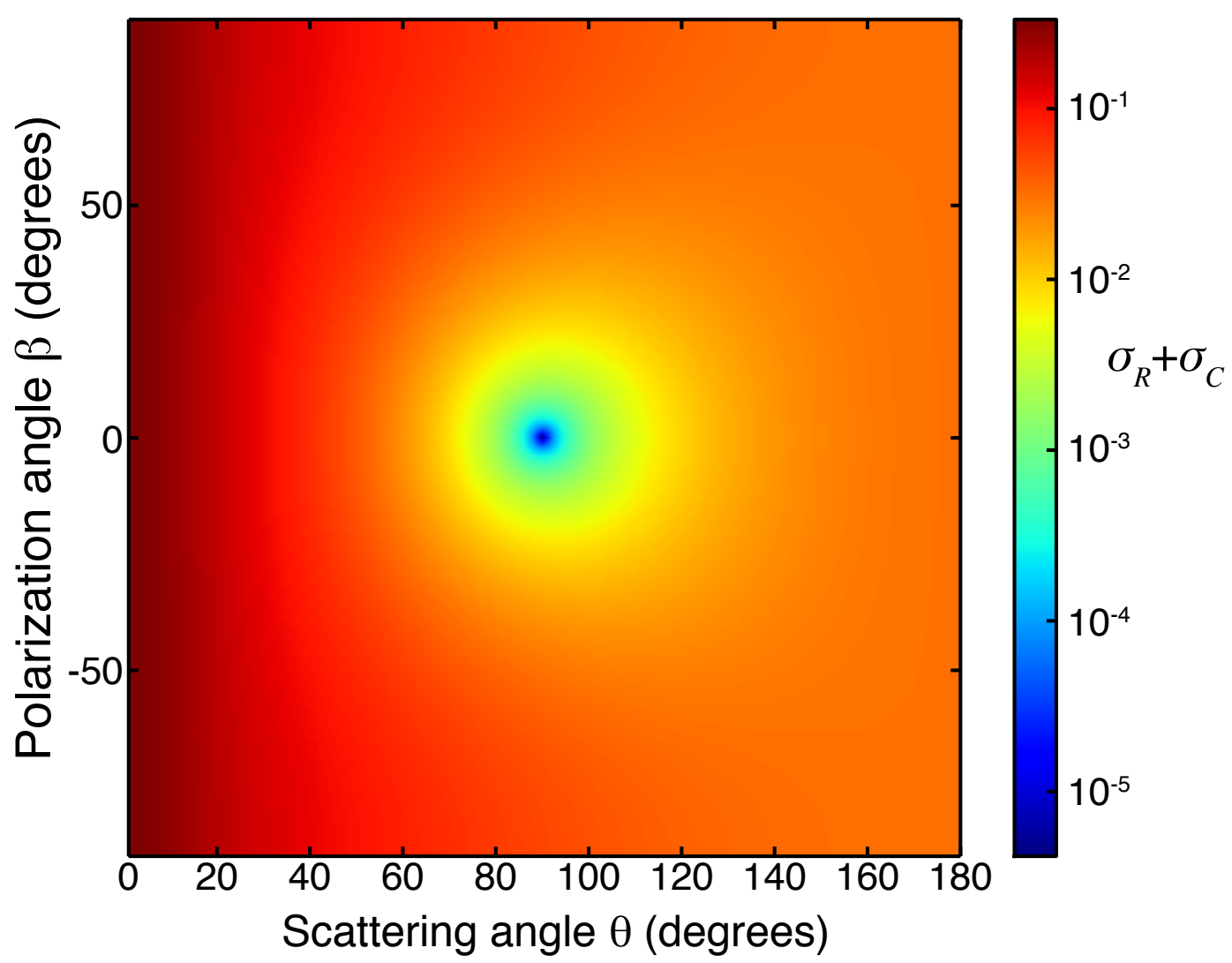

Figure 3: Differential cross section $\left(\sigma_{\mathrm{R}}+\sigma_{\mathrm{C}}\right)$ for Rayleigh and Compton scattering from silicon under polarized illumination with $E=12 \mathrm{KeV}$ X-rays. The scattering angle $\theta$ and the polarization angle $\beta$ are defined as the polar and azimuthal angles in the spherical coordinate system, with the $z$ axis pointing to the direction of the incident beam. Rayleigh scattering is strongly minimized at $90^{\circ}$ to the incident beam in the plane of polarization. Differential cross sections for scattering by other elements show the same pattern.

\subsection{Signal-to-noise ratio}

Our goal is to provide estimates on imaging particular elemental features in the presence of noise due to photon statistics and due to background signals. Following an approach outlined by Glaeser [40] and developed further by Sayre et al. [41, 42], our calculations are based on $\bar{n}$ photons incident, and then comparing measurements when a particular feature is present (in which case we measure a mean image intensity of $\bar{n} I_{p}$ photons, where $I_{\bullet}$ denotes the probability of getting a photon of a particular type), versus when the feature is absent (in which case we measure a mean image intensity of $\bar{n} I_{a}$ photons). Our signal is the difference between these two measurements. 


\subsubsection{Definition of $S / N$ and $P / B$}

One straightforward evaluation of the signal with respect to the background would be the peak-tobackground ratio $(\mathrm{P} / \mathrm{B})$, which is the ratio of the desired signal $\left(\bar{n} I_{p}-\bar{n} I_{a}\right)$ to the background signal $\left(\bar{n} I_{a}\right)$, or

$$
\mathrm{P} / \mathrm{B}=\frac{\bar{n} I_{p}-\bar{n} I_{a}}{\bar{n} I_{a}}
$$

However, a real spectrum is subject to statistical fluctuations, which blurs the difference between the featurepresent and -absent signals. We will assume that all noise sources are uncorrelated and can be described by a normal or Gaussian distribution. This is acceptably close to the correct Poisson distribution for the case of "shot noise" even when fewer than 10 photons are measured (see Supplementary). The combined error for comparing the two measurements is given by their root mean square sum. This leads immediately to a signal to noise ratio $(\mathrm{S} / \mathrm{N})$ of

$$
\begin{aligned}
\mathrm{S} / \mathrm{N} & =\frac{\left|\bar{n} I_{p}-\bar{n} I_{a}\right|}{\sqrt{\left(\sqrt{\bar{n} I_{p}}\right)^{2}+\left(\sqrt{\bar{n} I_{a}}\right)^{2}}} \\
& =\sqrt{\bar{n}} \frac{\left|I_{p}-I_{a}\right|}{\sqrt{I_{p}+I_{a}}}=\sqrt{\bar{n}} \Theta,
\end{aligned}
$$

where $\Theta=\left|I_{p}-I_{a}\right| / \sqrt{I_{p}+I_{a}}$ is the contrast parameter.

What leads to measured intensities? When one is measuring transmitted beams with background signal much smaller than "shot noise" (i.e., $I_{a} \ll \sqrt{I_{p}+I_{a}}$ ), Equation $(2)$ is sufficient. However, in elemental analysis based on X-ray emission (XRF, EPMA, and PIXE), one usually has an energy- or wavelength-dispersive spectrometer (EDS or WDS) to detect the signal corresponding to a particular element's characteristic fluorescent X-ray. In this case, we assume that the total intensity measured is the sum of a 'pure' feature intensity $\bar{n} I_{f}$ (such as a fluorescence signal), and a background intensity $\bar{n} I_{b}$ which might arise from other factors such as scattering of the incident beam. In this case, we can write the feature-present signal as $\bar{n} I_{p}=\bar{n} I_{f}+\bar{n} I_{b}$, while the feature-absent signal is simply $\bar{n} I_{a}=\bar{n} I_{b}$ so that Equation (2) can be written as

$$
\begin{aligned}
(\mathrm{S} / \mathrm{N})_{Z} & =\sqrt{\bar{n}} \frac{\left|I_{p}-I_{a}\right|}{\sqrt{I_{p}+I_{a}}}=\sqrt{\bar{n}} \frac{\left|\left(I_{f}+I_{b}\right)-I_{b}\right|}{\sqrt{\left(I_{f}+I_{b}\right)+I_{b}}} \\
& =\sqrt{\bar{n}} \frac{I_{f}}{\sqrt{I_{f}+2 I_{b}}}=\sqrt{\bar{n}} \Theta_{Z}
\end{aligned}
$$
cases where $I_{b} \ll I_{f}$, Equation (3) reduces to $(S / N)_{Z}=\sqrt{\bar{n} I_{f}}$ or $\bar{n}=(\mathrm{S} / \mathrm{N})_{Z}^{2} / I_{f}$, which is consistent with the simplifying assumption used by $\operatorname{Kirz}[15,16,17]$ that one needs to detect 25 fluorescence photons for elemental analysis with $S / N=5$.

\subsubsection{Quantification of the signal and the background}

One important problem in quantitative analysis of trace element concentrations is to identify and quantify the signal and the background. In order to do that, one needs to have reliable approximations of the back- 
ground to estimate the signal above the background. When dealing with real spectra from the experiment, one can use non-iterative background approximation methods like the three-window method [43, p. 397], interpolation, and polynomial fitting [44]. One may also use iterative methods like simple multiple-point smoothing [45], or more sophisticated ones like the Statistics-sensitive Non-linear Iterative Peak-clipping (SNIP) algorithm [46]. All of these methods can be problematic if inappropriate parameters (such as sampling width, or number of iterations) are chosen, or when the background is irregularly shaped.

Since we will deal primarily with analytically calculated spectra in this paper, in which every component contributing to the total signal is known, we are able to obtain the signal and the background without using any of the background approximation methods (this calculation includes detector response modeling as described in Section 3.4). Instead, we obtain the signal and the background by integrating the calculated fluorescence and total spectra within the full width at half maximum (FWHM) of the fluorescence peak (Figure 4). This will allow us to analyze the signal and the background independent of the spectral quantification methods used. However, we should note that when the energy resolution of the detector is poor or when there are multiple peaks piled up in the spectra, peak overlaps could happen; such cases are not addressed by our analytical method.

\subsubsection{Criteria on minimal detectable limit}

We comment briefly on the desired signal-to-noise ratio. In a review of several studies of visual perception [47], Rose concluded that the human eye can reliably discern test pattern features in low light conditions when the signal to noise ratio is 5 or better, and $S / N=5$ is usually referred to as the "Rose criterion." However, Rose also referred to unpublished work by Schade that suggested $S / N=3$ allowed patterns to be discerned on early television pictures, and very low $\mathrm{S} / \mathrm{N}$ values are routinely accepted when combining multiple images in single particle electron microscopy [48, Fig. 5]. We use $(S / N)=3$ for our minimum exposure calculations.

\subsection{Detector geometries}

In principle, one might hope to locate a fluorescence detector at any position in the $4 \pi$ solid angle surrounding the sample. In practise, three positions are most commonly used: the $0^{\circ}$ or forward-scattering position, the $180^{\circ}$ or back-scattering position, and the $90^{\circ}$ or sideways position as shown in Figure 2.

The $90^{\circ}$ geometry is most commonly used due to the minimized (elastic and inelastic) scattering background, as noted above. However, since the sample plane is always put at a non-perpendicular angle to the incident beam (often $45^{\circ}$ ), this geometry suffers from reduced (and asymmetric) spatial resolution from an elongated beam footprint and increased self-absorption artifacts, due to oblique incident and take-off angles. Moreover, the tilted sample also makes it hard to put the detector close to the sample surface without the 


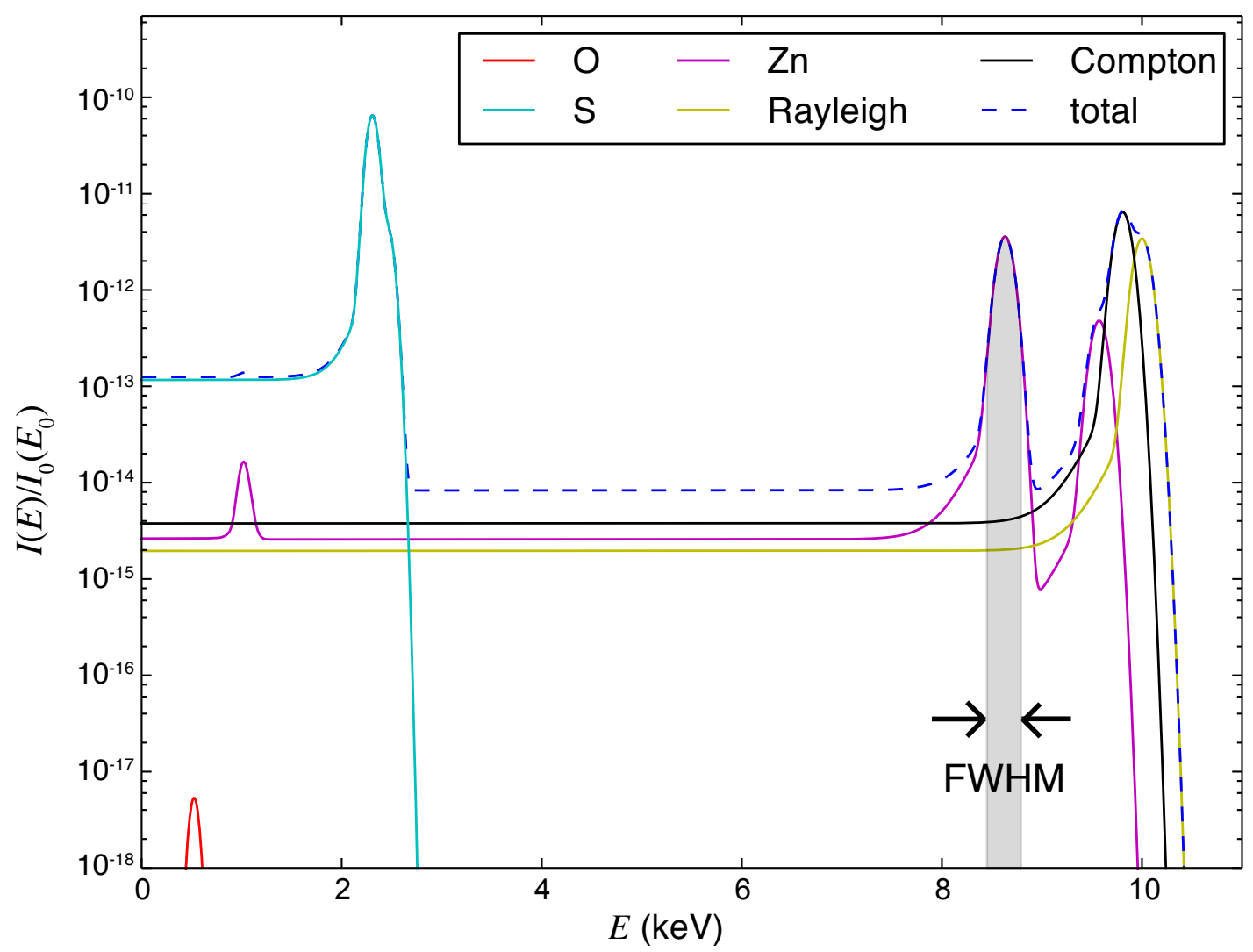

Figure 4: Calculated energy-dispersive spectra of the modeled "bio" sample. The incident energy is $E=10 \mathrm{KeV}$. A detector at $90^{\circ}$ with collection semi-angle $\phi=5^{\circ}$ is assumed. Spectral components from fluorescence of constituent elements and Rayleigh and Compton scattering are shown using different colors, based on a model of detector response described in Section 3.4. The feature signal and the background are obtained by integrating the calculated fluorescence and total spectra within the FWHM of the fluorescence peak. The range of integration is marked in gray for the $\mathrm{Zn} K \alpha_{1}$ peak.

sample hitting the detector during scanning, thus hindering the scanning of large planar samples (such as paintings and other cultural heritage specimens) and the enlargement of the collection angle.

Recently, the $180^{\circ}$ geometry has been used with the 384-element Maia detector [31]. This geometry is advantageous because it has normal incidence and take-off angles, so that the focal spot size of the illuminating source is not elongated when projected onto the sample, and large planar samples can be scanned parallel to the sample surface. In addition, the detector can be placed very close to the sample surface to obtain a large collection angle, and there is no fluorescence self-absorption from the topmost layer of the sample facing the incident beam. On the other hand, since the detector has to be placed between the sample and the focusing optics (such as a Fresnel zone plate and associated Order Sorting Aperture or OSA), this geometry limits the room for the detector especially when, at very high spatial resolution, the focal length of the focusing optics is small. In addition, a hole in the center of the detector is required in 


\begin{tabular}{|l|c|c|c|}
\hline Detector position & $0^{\circ}$ & $90^{\circ}$ & $180^{\circ}$ \\
\hline Scattering background & largest & minimized & large \\
\hline Incident and take-off angles & normal $\left(90^{\circ}\right)$ & oblique (often $\left.45^{\circ}\right)$ & normal $\left(90^{\circ}\right)$ \\
\hline Scanning direction & parallel & non-parallel & parallel \\
\hline Space for detector & large & large & limited \\
\hline Detector-sample distance & no restriction & large & small \\
\hline $\begin{array}{l}\text { Attenuation artifacts } \\
\text { (homogeneous samples) }\end{array}$ & $\begin{array}{l}\text { small (thin sample) } \\
\text { large (thick sample) }\end{array}$ & medium & small \\
\hline
\end{tabular}

\section{Simulations of XRF spectra}

In order to better understand the nature of the signal and the scattering background from the specimen in the XRF spectra, we simulated the XRF spectra of our two representative samples using an analytical method which we now describe. This approach complements more detailed Monte Carlo calculations such as those provided by the XMI-MSIM $[27,50]$ package, which includes plural scattering and the mutual fluorescence enhancement effect. However, Monte Carlo methods are usually much more time-consuming. We will show in Section 3.5 that our analytical method gives adequately accurate results using less than one tenth of the computation time. Moreover, as described in Section 2.1.2, the analytical method provides spectra with separately categorized signal components, which makes for easier quantitative evaluation of signal versus background components in the final result. The source code for XRF spectra calculation using our analytical method is published online[51]. 


\subsection{The layered sample model}

We use a layered model for the specimen (Figure 5). Beam is incident on the specimen at an angle $\Psi$. The scattered or emitted beam exits the specimen with a positive take-off angle of $\Psi^{\prime}>0$ with respect

Scattering and fluorescence signals are calculated in thin layers with element thickness $d t$, and integrated through all layers of the sample, with the attenuation of the incident and emergent beams taken into account.

\subsection{Scattering and fluorescence cross sections}

The strength of the interactions between photons and matter (with thickness $d t$ ) can be represented by their cross-sections, which are defined as the number of photons interacting with one atom per unit time per unit incident photon flux $(\Phi)$. Note that the incident beam intensity $I=\Phi A$, (where A is the cross-sectional area of the beam,) so

$$
\sigma=-\frac{d I}{N \Phi}=-\frac{A d I}{N I},
$$

where $N$ is the number of atoms in the interacting matter, and $-d I$ is the intensity of the photons that interact with them. To obtain the angular distribution of the emergent photons after interaction, we have to use the differential cross section, which is defined as the number of photons going into a solid angle $d \Omega$ per unit time per unit incident photon flux, divided by the solid angle $d \Omega$, or

$$
\frac{d \sigma}{d \Omega}=-\frac{A d I}{N I d \Omega} .
$$

Equation (4) can also be rewritten as

$$
\frac{d I}{I}=-\left(\frac{N}{A d t}\right) \sigma d t=-\left(\frac{N}{V}\right) \sigma d t=-\left(\frac{\rho N_{A}}{M}\right) \sigma d t=-n \sigma d t=-\mu d t
$$




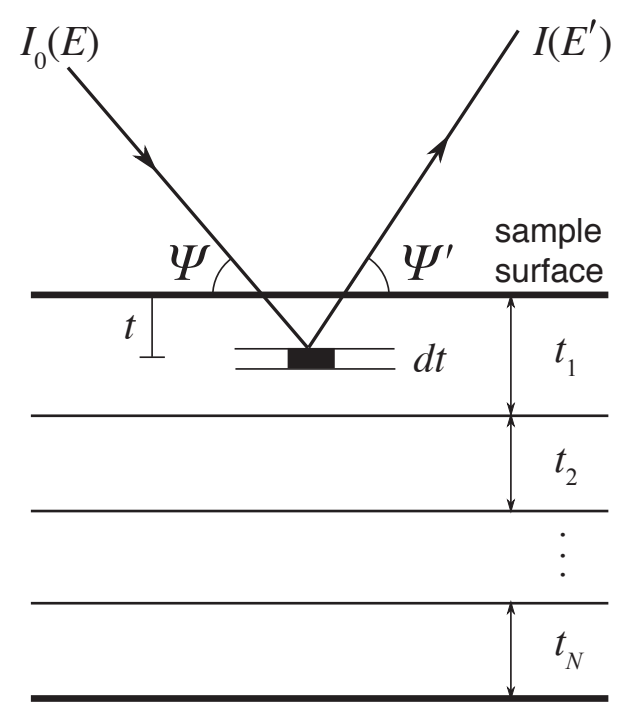

(a) Backwards geometry for layered samples.

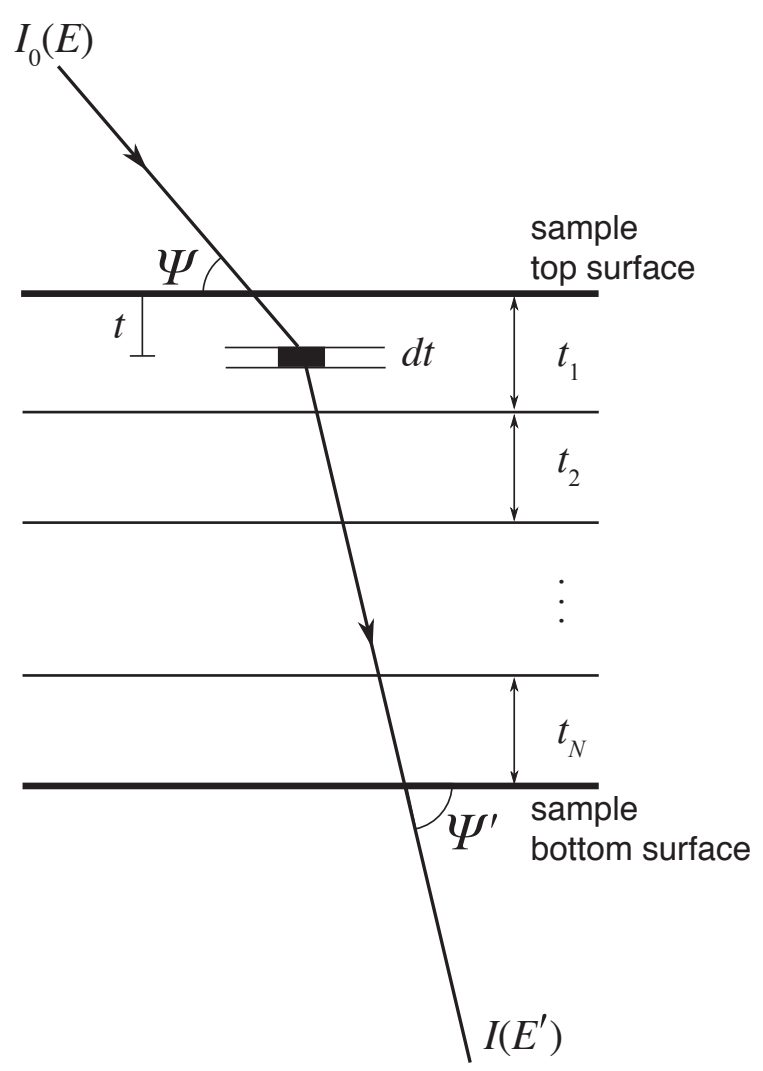

(b) Forwards geometry for layered samples.

Figure 5: The layered sample model for the specimen in spectra calculations. The incident beam enters the specimen at an angle $\Psi$, and the scattered or fluorescent signal is emitted at an angle $\Psi^{\prime}$ which is positive in the backwards geometry and negative in the forwards geometry. Scattering and fluorescence are calculated in layers with thickness $d t$, and integrated through all layers of the sample. Attenuation of the incident and emergent beams are taken into account.

where $n=N / V$ is the number density of the scatterer, $N_{A}$ is Avogadro's number, $\rho$ is the mass density, and $M$ is the molar mass. The linear attenuation coefficient is

$$
\mu=\sigma n=\sigma \frac{N_{A} \rho}{M} .
$$

Because the cross section is unaffected by the density of the interacting medium, it is convenient to define the mass attenuation coefficient [52, p. 20] [53, p. 200]

$$
\frac{\mu}{\rho}=\frac{\sigma N_{A}}{M} .
$$

The differential form of Equation (8) can be directly deduced from Equations (6) and (7).

In our calculations, only three types of interactions are considered: elastic (Rayleigh), inelastic (Compton) scattering, and X-ray fluorescence. The intensities of the Rayleigh and Compton scattered photons are calculated from their differential cross sections for plane-polarized radiation, which are deduced from 
the Thomson and Klein-Nishina [35, 36] formulas combined with the atomic form factor $f(x, Z)$ and the incoherent scattering factor $s(x, Z)$, respectively. The differential cross section of Rayleigh scattering is given by

$$
\frac{d \sigma_{\mathrm{R}}}{d \Omega}=\frac{d \sigma_{\mathrm{Th}}}{d \Omega}[f(x, Z)]^{2},
$$

while for Compton scattering we have

$$
\frac{d \sigma_{\mathrm{C}}}{d \Omega}=\frac{d \sigma_{\mathrm{KN}}}{d \Omega} s(x, Z) .
$$

For plane-polarized radiation, the Thomson and Klein-Nishina differential cross sections are written as

$$
\frac{d \sigma_{\mathrm{Th}}}{d \Omega}=\frac{r_{e}^{2}}{2}\left(\cos ^{2} \beta \cos ^{2} \theta+\sin ^{2} \beta\right),
$$

and

$$
\frac{d \sigma_{\mathrm{KN}}}{d \Omega}=\frac{r_{e}^{2}}{2}\left(\frac{E^{\prime}}{E}\right)^{2}\left(\frac{E^{\prime}}{E}+\frac{E}{E^{\prime}}-2 \sin ^{2} \theta \cos ^{2} \beta\right),
$$

where $E$ and $E^{\prime}$ are the energies of the incident and scattered photons, and $\theta$ and $\beta$ are the scattering angle and the polarization angle, defined as the polar and azimuthal angles of the scattered photon in the spherical coordinates where the $z$ axis points in the direction of the incident beam, and $\beta=0$ is along the polarization vector of the incident beam. Note that $\Psi$ and $\Psi^{\prime}$ are different from the scattering angle $\theta$, and polarization angle $\beta$.

The atomic form factor $f(x, Z)$ and incoherent scattering factor $s(x, Z)$ can be obtained from X-ray scattering databases such as Xraylib [54], where

$$
x=\frac{\sin \left(\frac{\theta}{2}\right)}{\lambda}
$$

is the momentum transfer. The XRF cross section $\sigma_{\mathrm{YX}}(E)$ of the fluorescence line associated with transition from electron shell $\mathrm{X}$ to shell $\mathrm{Y}$ in an atom (under an incident radiation with energy $E$ ) is given by

$$
\sigma_{\mathrm{YX}}(E)=\sigma_{\mathrm{Y}}(E) \omega_{\mathrm{Y}} F_{\mathrm{YX}}
$$

where $\sigma_{\mathrm{Y}}(E)$ is the partial photoelectric absorption cross section of shell $\mathrm{Y}, \omega_{\mathrm{Y}}$ is the fluorescence yield of shell $\mathrm{Y}$, and $F_{\mathrm{YX}}$ is the transition probability from shell $\mathrm{Y}$ to shell $\mathrm{X}$.

One can use tabulated data [55] for $\sigma_{\mathrm{Y}}(E), \omega_{\mathrm{Y}}$, and $F_{\mathrm{YX}}$, and then combine them to get $\sigma_{\mathrm{YX}}(E)$. Recently, Schoonjans et al. calculated the partial fluorescence cross sections of specific fluorescence lines based on [55]. Full cascade effects are included in their calculations. The results are available in the online database Xraylib [54]. In our calculations, we used the Kissel partial fluorescence cross sections with full cascade from Xraylib.

For a compound,

$$
\left(\frac{\mu}{\rho}\right)_{\text {compound }}=\sum_{i} W_{i}\left(\frac{\mu}{\rho}\right)_{i},
$$

where $W_{i}$ is the weight fraction of the $i$ th element in the compound. 


\subsection{Correlation between intensity and concentration}

The intensities of the X-ray fluorescence emissions are calculated using the Sherman equation [56]. Based on the Sherman equation, to the first order approximation (no chain effect), the intensity of the characteristic fluorescence photons from a single layer sample reaching a solid angle element $d \Omega$ in reflection geometry is

$$
d I_{\mathrm{YX}}\left(E^{\prime}\right)=\frac{I_{0}(E) \mu_{\mathrm{YX}}\left(E, E^{\prime}\right)}{4 \pi\left[\mu_{\mathrm{total}}(E)+\frac{\sin \Psi}{\sin \Psi^{\prime}} \mu_{\mathrm{total}}\left(E^{\prime}\right)\right]} d \Omega\left\{1-e^{-\left[\frac{\mu_{\mathrm{total}}(E)}{\sin \Psi}+\frac{\mu_{\mathrm{total}}\left(E^{\prime}\right)}{\sin \Psi^{\prime}}\right] t}\right\}
$$

$$
\text { radiation, } \Psi \text { and } \Psi^{\prime} \text { are the incident and emergent angles as defined in Figure } 5, \mu_{\text {total }}=\mu_{\text {Rayleigh }}+
$$
$\mu_{\text {Compton }}+\mu_{\mathrm{PE}}$ is the total attenuation coefficient (PE stands for photoelectric absorption), and $t$ is the thickness of the single layer.

Equation (14) can also be generalized to scattering, if we substitute $\mu_{\mathrm{YX}}\left(E, E^{\prime}\right) / 4 \pi$ by the differential attenuation coefficient for scattering. The intensities of the Rayleigh and Compton scattered photons are given by

$$
d I_{\mathrm{R}}(E)=\frac{I_{0}(E)}{\left(1+\frac{\sin \Psi}{\sin \Psi^{\prime}}\right) \mu_{\mathrm{total}}(E)}\left[\frac{d \mu_{\mathrm{R}}(E)}{d \Omega}\right] d \Omega\left[1-e^{-\left(\frac{1}{\sin \Psi}+\frac{1}{\sin \Psi^{\prime}}\right) \mu_{\mathrm{total}}(E) t}\right]
$$

and

$$
d I_{\mathrm{C}}\left(E^{\prime}\right)=\frac{I_{0}(E)}{\left[\mu_{\text {total }}(E)+\frac{\sin \Psi}{\sin \Psi^{\prime}} \mu_{\text {total }}\left(E^{\prime}\right)\right]}\left[\frac{d \mu_{\mathrm{C}}(E)}{d \Omega}\right] d \Omega\left\{1-e^{-\left[\frac{\mu_{\text {total }}(E)}{\sin \Psi}+\frac{\mu_{\text {total }}\left(E^{\prime}\right)}{\sin \Psi^{\prime}}\right] t}\right\} .
$$

Note that here $d \mu_{\mathrm{R}}(E) / d \Omega$ and $d \mu_{\mathrm{C}}(E) / d \Omega$ are dependent on $\theta$ and $\beta$, which accounts for the angular dependence of $d I_{\mathrm{R}}$ and $d I_{\mathrm{C}}$.

We have extended Equations (14) to (16) to layered samples and the transmission geometry to suit our sample models. In case of a layered specimen, the XRF intensity generated from the $m$ th layer in the reflection geometry is

$$
\left.d I_{\mathrm{YX}}^{(m)}\left(E^{\prime}\right)=\frac{I_{0}(E) \mu_{\mathrm{YX}}^{(m)}\left(E, E^{\prime}\right)}{4 \pi\left[\mu_{\text {total }}^{(m)}(E)+\frac{\sin \Psi}{\sin \Psi^{\prime}} \mu_{\text {total }}^{(m)}\left(E^{\prime}\right)\right]} d \Omega\left\{1-e^{-\left[\frac{\mu_{\text {total }}^{(m)}(E)}{\sin \Psi}+\frac{\mu_{\text {total }}^{(m)}\left(E^{\prime}\right)}{\sin \Psi^{\prime}}\right]}\right] t_{m}\right\} A_{\text {in }}^{(m)} A_{\text {out }}^{(m)}
$$

where

$$
\begin{aligned}
& A_{\mathrm{in}}^{(m)}=\prod_{i=1}^{m-1} e^{-\frac{\mu_{\mathrm{total}}^{(i)}(E)}{\sin \Psi} t_{i}} \\
& A_{\mathrm{out}}^{(m)}=\prod_{i=1}^{m-1} e^{-\frac{\mu_{\text {total }}^{(i)}\left(E^{\prime}\right)}{\sin \Psi^{\prime}} t_{i}}
\end{aligned}
$$

are the attenuation factors for the incident radiation going in through the $m-1$ layers above the $m$ th layer, 
and the emergent radiation going out through those $m-1$ layers, which can be combined into one factor

$$
\begin{aligned}
A^{(m)} & =A_{\mathrm{in}}^{(m)} A_{\text {out }}^{(m)} \\
& =\prod_{i=1}^{m-1} e^{-\left[\frac{\mu_{\text {total }}^{(i)}(E)}{\sin \Psi}+\frac{\mu_{\text {total }}^{(i)}\left(E^{\prime}\right)}{\sin \Psi^{\prime}}\right] t_{i}} \\
& =e^{-\sum_{i=1}^{m-1}\left[\frac{\mu_{\text {total }}^{(i)}(E)}{\sin \Psi}+\frac{\mu_{\text {total }}^{(i)}\left(E^{\prime}\right)}{\sin \Psi^{\prime}}\right]} t_{i}
\end{aligned}
$$

In the transmission geometry, the attenuation of the fluorescence photons generated at $t$ in a homogeneous layer with thickness $t_{m}$ is

$$
e^{-\frac{\mu_{\mathrm{total}}\left(E^{\prime}\right)}{\left|\sin \Psi^{\prime}\right|}\left(t_{m}-t\right)}=e^{-\frac{\mu_{\mathrm{total}}\left(E^{\prime}\right)}{\sin \Psi^{\prime}}\left(t-t_{m}\right)} .
$$

Note that $\Psi^{\prime}<0$ in the transmission geometry, so $\left|\sin \Psi^{\prime}\right|=-\sin \Psi^{\prime}$. Therefore, the Sherman equation for the transmission geometry is

$$
\begin{aligned}
d I_{\mathrm{YX}}\left(E^{\prime}\right) & =\frac{I_{0}(E) \mu_{\mathrm{YX}}\left(E, E^{\prime}\right)}{4 \pi \sin \Psi} d \Omega \int_{0}^{t_{m}} e^{-\left[\frac{\mu_{\mathrm{total}}(E)}{\sin \Psi} t+\frac{\mu_{\mathrm{total}}\left(E^{\prime}\right)}{\sin \Psi^{\prime}}\left(t-t_{m}\right)\right]} d t \\
& =\frac{I_{0}(E) \mu_{\mathrm{YX}}\left(E, E^{\prime}\right)}{4 \pi\left[\mu_{\text {total }}(E)+\frac{\sin \Psi}{\sin \Psi^{\prime}} \mu_{\text {total }}\left(E^{\prime}\right)\right]} d \Omega\left[e^{\frac{\mu_{\mathrm{total}}\left(E^{\prime}\right)}{\sin \Psi^{\prime}} t}-e^{-\frac{\mu_{\mathrm{total}}(E)}{\sin \Psi} t}\right] .
\end{aligned}
$$

In a layered sample, the XRF intensity from layer $m$ is given by

$$
d I_{\mathrm{YX}}^{(m)}\left(E^{\prime}\right)=\frac{I_{0}(E) \mu_{\mathrm{YX}}^{(m)}\left(E, E^{\prime}\right)}{4 \pi\left[\mu_{\text {total }}^{(m)}(E)+\frac{\sin \Psi}{\sin \Psi^{\prime}} \mu_{\text {total }}^{(m)}\left(E^{\prime}\right)\right]} d \Omega\left[e^{\frac{\mu_{\text {total }}^{(m)}\left(E^{\prime}\right)}{\sin \Psi^{\prime}} t_{m}}-e^{-\frac{\mu_{\text {total }}^{(m)}(E)}{\sin \Psi} t_{m}}\right] A_{\mathrm{in}}^{(m)} A_{\text {out }}^{(m)}
$$

where

$$
\begin{aligned}
& A_{\mathrm{in}}^{(m)}=\prod_{i=1}^{m-1} e^{-\frac{\mu_{\mathrm{total}}^{(i)}(E)}{\sin \Psi} t_{i}} \\
& A_{\mathrm{out}}^{(m)}=\prod_{i=m+1}^{N} e^{-\frac{\mu_{\mathrm{total}}^{(i)}\left(E^{\prime}\right)}{\left|\sin \Psi^{\prime}\right|} t_{i}}=\prod_{i=m+1}^{N} e^{\frac{\mu_{\text {total }}^{(i)}\left(E^{\prime}\right)}{\sin \Psi^{\prime}} t_{i}}
\end{aligned}
$$

are the attenuation factors for the incident radiation going in through the $m-1$ layers above the $m$ th layer, and the emergent radiation going out through the $N-m$ layers under it. Combining similar factors, we arrive at

$$
\begin{aligned}
& d I_{\mathrm{YX}}^{(m)}\left(E^{\prime}\right)=\frac{I_{0}(E) \mu_{\mathrm{YX}}^{(m)}\left(E, E^{\prime}\right)}{4 \pi\left[\mu_{\text {total }}^{(m)}(E)+\frac{\sin \Psi}{\sin \Psi^{\prime}} \mu_{\text {total }}^{(m)}\left(E^{\prime}\right)\right]} d \Omega\left\{1-e^{-\left[\frac{\mu_{\text {total }}^{(m)}(E)}{\sin \Psi}+\frac{\mu_{\text {total }}^{(m)}}{\left.\sin \Psi^{\prime}\right)}\right] t_{m}}\right\} e^{\frac{\mu_{\text {total }}^{(m)}\left(E^{\prime}\right)}{\sin \Psi^{\prime}} t_{m}} A_{\text {in }}^{(m)} A_{\text {out }}^{(m)} \\
& =\frac{I_{0}(E) \mu_{\mathrm{YX}}^{(m)}\left(E, E^{\prime}\right)}{4 \pi\left[\mu_{\text {total }}^{(m)}(E)+\frac{\sin \Psi}{\sin \Psi^{\prime}} \mu_{\text {total }}^{(m)}\left(E^{\prime}\right)\right]} d \Omega\left\{1-e^{-\left[\frac{\mu_{\text {total }}^{(m)}}{\sin \Psi}+\frac{\mu_{\text {total }}^{(m)}\left(E^{\prime}\right)}{\sin \Psi^{\prime}}\right]} t_{m}\right\} A^{(m)}
\end{aligned}
$$

where

$$
\begin{aligned}
A^{(m)} & =e^{\frac{\mu_{\text {total }}^{(m)}\left(E^{\prime}\right)}{\sin \Psi^{\prime}} t_{m}} A_{\mathrm{in}}^{(m)} A_{\text {out }}^{(m)} \\
& =\left[\prod_{i=1}^{m-1} e^{-\frac{\mu_{\text {total }}^{(i)}(E)}{\sin \Psi} t_{i}}\right]\left[\prod_{i=m}^{N} e^{\frac{\mu_{\text {total }}^{(i)}\left(E^{\prime}\right)}{\sin \Psi^{\prime}} t_{i}}\right]
\end{aligned}
$$


Adding up the contributions from all the layers in the sample, we have a total intensity of XRF line YX of

$$
d I_{\mathrm{YX}}\left(E^{\prime}\right)=\sum_{m=0}^{N} d I_{\mathrm{YX}}^{(m)}\left(E^{\prime}\right)
$$

The intensities of Rayleigh and Compton scattering can be deduced accordingly.

\subsection{Detector response}

As discussed in Section 2.1.2, one advantage of working with an analytical model is that one can easily separate out signal versus background components if one has a model of the response of the energy dispersive detector. We assume that photons incident on the detector are filtered with a $24 \mu \mathrm{m}$ thick Be window, as is typically installed. Perfect spectral lines in the calculated theoretical spectra are then convolved with the detector response function, where we will use the same notation as [44]. The detector response is described by a modified Gaussian function of

$$
P\left(E_{i}, E_{j}\right)=G\left(E_{i}, E_{j}\right)+f_{S} S\left(E_{i}, E_{j}\right)+f_{T} T\left(E_{i}, E_{j}\right)
$$

where

$$
G\left(E_{i}, E_{j}\right)=\frac{\text { GAIN }}{s \sqrt{2 \pi}} \exp \left[-\frac{\left(E_{j}-E_{i}\right)^{2}}{2 s^{2}}\right]
$$

is the Gaussian function which describes the broadening of the peak due to limited energy resolution of the detector. The additional terms are a step function

$$
S\left(E_{i}, E_{j}\right)=\frac{\text { GAIN }}{s \sqrt{2 E_{j}}} \operatorname{erfc}\left[\frac{E_{i}-E_{j}}{s \sqrt{2}}\right]
$$

and a tailing function

$$
T\left(E_{i}, E_{j}\right)=\frac{\text { GAIN }}{2 \gamma s \exp \left[-1 /\left(2 \gamma^{2}\right)\right]} \exp \left[\frac{E_{i}-E_{j}}{\gamma s}\right] \operatorname{erfc}\left[\frac{E_{i}-E_{j}}{s \sqrt{2}}+\frac{1}{\gamma \sqrt{2}}\right]
$$

where $\gamma$ denotes the broadening of the exponential tail. The step and tailing functions account for the incomplete charge collection of the detector. GAIN is the detector channel gain in $\mathrm{eV} /$ channel, $E_{j}$ the energy (in $\mathrm{eV}$ ) of the X-ray line, and $E_{i}$ the energy (in $\mathrm{eV}$ ) of the $i$ th channel of the detector. The peak width $s$ is given by

$$
s^{2}=\left(\frac{\mathrm{NOISE}}{2.3548}\right)^{2}+3.58 \mathrm{FANO} E_{j}
$$

where NOISE is the electronic contribution to the peak width (typically $80-100 \mathrm{eV} \mathrm{FWHM)} \mathrm{with} \mathrm{the} \mathrm{factor}$ $2 \sqrt{2 \ln 2} \approx 2.3548$ converting FWHM to $\sigma$; FANO is the Fano factor $(\sim 0.114)$, and 3.58 is the energy (in $\mathrm{eV})$ required to produce an electron-hole pair in silicon [44, p. 282]. The factors $f_{S}=0.03$ and $f_{T}=0.02$ describe the fractions of photon intensities that arrive in the step and the tail, respectively (Figure 6), and again these values are for silicon [44, p. 282]. 


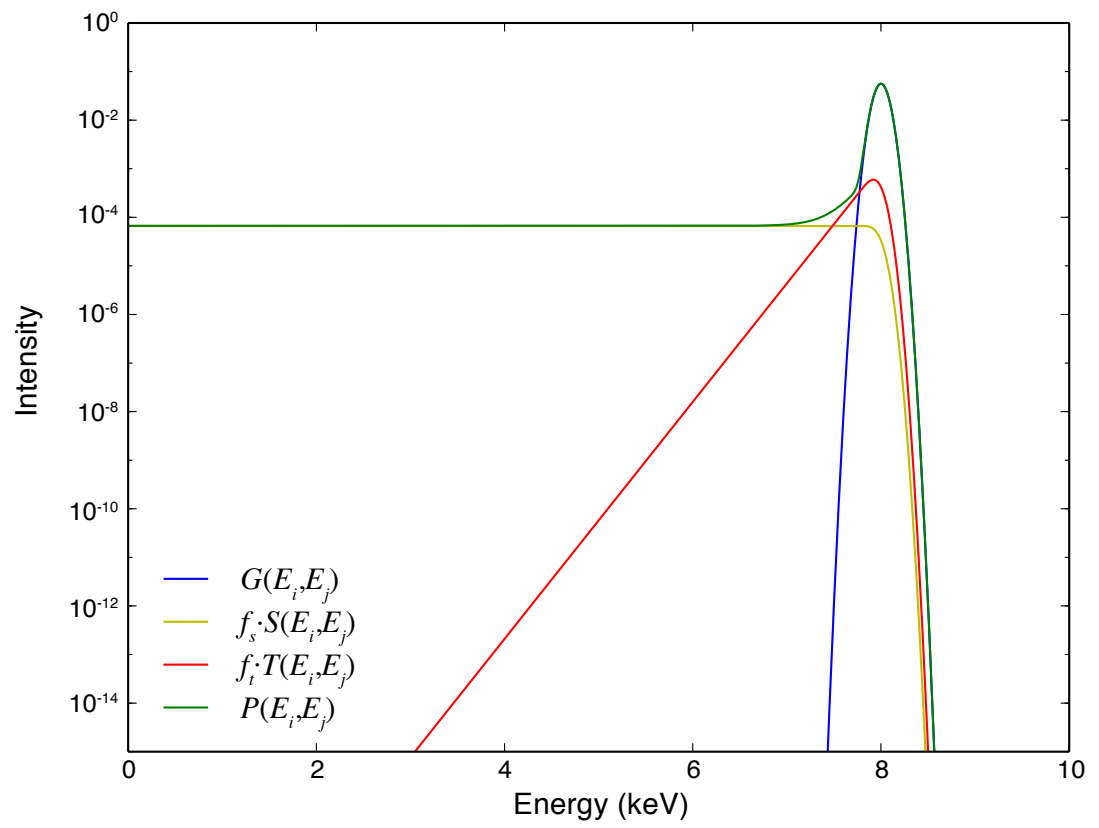

Figure 6: Detector response of an ideal peak at $8 \mathrm{KeV}$ with unit height, and the contributions of its constituent components. This calculation used parameters (described in Section 3.4) of GAIN=10, NOISE $=100$, FANO $=0.114, \gamma=2.5, f_{S}=0.03$, and $f_{T}=0.02$. These parameters will be used for all of the simulations described in this paper.

\subsection{Test of simulation against experiment}

In order to verify the validity of our simulations, we did a simple test experiment and compared the results with our simulations. We used AXO standard RF4-100-S1749 (AXO DRESDEN GmbH), which consists of 7 elements ( $\mathrm{Pb}, \mathrm{La}, \mathrm{Pd}, \mathrm{Mo}, \mathrm{Cu}, \mathrm{Fe}, \mathrm{Ca}$ ) deposited on a silicon nitride membrane window supported by a silicon frame. The silicon nitride membrane is $10 \mathrm{~nm}$ thick and $5 \mathrm{~mm}$ square, and the silicon frame is $10 \mathrm{~mm}$ square with $200 \mu \mathrm{m}$ thickness. The standard deposited on top of the window is $\sim 10$ atomic layers $(\sim 3 \mathrm{~nm})$. The test experiments were conducted at beamline 2-ID-D at the Advanced Photon Source at Argonne National Laboratory. The incident photon energy was set to $10.1 \mathrm{KeV}$, and was focused using a Fresnel zone plate. The sample is positioned so that the sample plane is $75^{\circ}$ to the incident beam $\left(15^{\circ}\right.$ to the detector at $90^{\circ}$ ). A one element Vortex-EM (Hitachi High-Technologies Science America, Inc.) silicon drift X-ray detector was positioned $30 \mathrm{~mm}$ away from the sample, and at 50, 90, and $124^{\circ}$ to the incident beam. The detector has an active area of $50 \mathrm{~mm}^{2}$, subtending a collection semi-angle of $\sim 6.7^{\circ}$ at $30 \mathrm{~mm}$ from the sample. Background signals with only the Al stick holding the sample were recorded under the same conditions and subtracted from the spectra with the standard present. Calculated spectra were compared with the experimental data, as shown in Figure 7. Spectra from Monte Carlo simulations using the XMIMSIM [27] package are also shown alongside. Attenuation by the air was included in both the calculation 
and the Monte Carlo simulation. The calculated and simulated spectra were scaled with the experimental data using the Fe K $\alpha$ peak. The spectra from calculation match very well with the Monte Carlo simulation results, except for some discrepancies in the Compton peaks and the peaks around $3 \mathrm{KeV}$. The former is due to the exclusion of Compton broadening profile in the analytical calculation; the latter is caused by the $\mathrm{Si}$ escape peaks from the $\mathrm{Ca} K \alpha$ peaks, which is neglected in the analytical calculation. In addition, the higher estimated background by the Monte Carlo method also adds up to the higher counts near $3 \mathrm{KeV}$. Both the Monte Carlo and the analytical methods give an underestimation of the $\mathrm{Si}$ and the scattering peaks. This may be caused by the extra scattering and fluorescence from the $\mathrm{Si}$ frame holding the $\mathrm{Si}_{3} \mathrm{~N}_{4}$ membrane. The experimental scattering peaks in the $90^{\circ}$ case are much larger than estimated either in our simulation or in the Monte Carlo calculation. That may be explained by the partial polarization of the X-ray source, which has a finite polarization vector in the direction perpendicular to the major polarization plane, and cannot be minimized by scattering at $90^{\circ}$ [57]. Moreover, the secondary air scattering of the photons scattered by the sample, which is not included in the measured background spectrum, can also introduce non- $90^{\circ}$ scattering that increases the scattering peaks significantly. (Moving the detector $2^{\circ}$ off $90^{\circ}$ does not change the scattering peak significantly). In spite of this one minor inconsistency, the overall agreement between our simulations, Monte Carlo calculations, and the real experimental data is quite satisfactory.

\section{Results on model specimens}

With our simulation results validated as described in Section 3.5, we then applied our approach to the two model specimens described in Section 1 . We considered detectors positioned at $0^{\circ}, 90^{\circ}$, and $180^{\circ}$ to the incident beam with collection semi-angle $\phi$. The detector is assumed to be in the major polarization plane of the incident radiation, which is the horizontal plane in the case of a synchrotron radiation source with vertical magnetic field directions.

The collection semi-angle $\phi$ of the detector is defined as the angle between the center and the edge of the detector viewing from the beam spot. The geometry of the detector at $90^{\circ}$ used in our calculations is slightly different from the ones at $0^{\circ}$ and $180^{\circ}$. If we use a spherical coordinate system with the $z$ axis pointing to the direction of the incident beam, the angle range subtended by the detector with $\phi=x^{\circ}$ is given in Table 2, where $\theta$ and $\beta$ are the scattering and polarization angles as defined previously in Equations (11) and (12). A plot of the solid angles subtended by detectors centered at $90^{\circ}$ versus $0^{\circ}$ and $180^{\circ}$ as a function of the collection semi-angle $\phi$ is shown in Figure 8.

For the "bio" sample, we kept the $0.02 \mu \mathrm{m}$ thick protein/zinc layer constant and sandwiched it in water layers to yield an overall sample thickness of $0.22 \mu \mathrm{m}, 2.02 \mu \mathrm{m}, 20.02 \mu \mathrm{m}$, and $200.02 \mu \mathrm{m}$. As we increase the thickness of the water layers, the background signal (from the tails of the scattering peaks due to incomplete charge collection) also increases. The $\mathrm{S}$ peak at around $2 \mathrm{keV}$ decreases due to increased absorption by 
Table 2: Angle range subtended by detectors in $0^{\circ}$ (forward-scattering), $90^{\circ}$ (sideways), and $180^{\circ}$ (back-scattering) geometries, with collection semi-angle $\phi=x^{\circ}$. Here $\theta$ is the polar angle or the scattering angle, and $\beta$ the azimuthal angle or the polarization angle.

\begin{tabular}{|l|c|c|c|}
\hline Detector center & $0^{\circ}$ & $90^{\circ}$ & $180^{\circ}$ \\
\hline$\theta$ range & $0^{\circ}-x^{\circ}$ & $(90-x)^{\circ}-(90+x)^{\circ}$ & $(180-x)^{\circ}-180^{\circ}$ \\
\hline$\beta$ range & $0^{\circ}-360^{\circ}$ & $-x^{\circ}-x^{\circ}$ & $0^{\circ}-360^{\circ}$ \\
\hline Shape & circular & semi-square & circular \\
\hline
\end{tabular}

the water layers, but the Zn peak with a much higher energy ( $>8 \mathrm{keV}$ ) does not suffer very much from self-absorption (Figure 9). If we keep the thickness of the water layers constant and increase the collection semi-angle $\phi$, both the scattering background and the feature XRF peak will increase (Figure 10). Spectra calculated with the other two detector geometries and for the "chip" sample with varying Si thickness $(0.5 \mu \mathrm{m}, 5 \mu \mathrm{m}, 50 \mu \mathrm{m}$ and $500 \mu \mathrm{m})$ show the same trend (see Supplementary). Comparing these spectra, we see that the background signal collected by a detector at $90^{\circ}$ is always smaller than by a detector of the same collection semi-angle at $180^{\circ}$, and even smaller when compared to $0^{\circ}$. While the signal levels are comparable for the sideways and back-scattering detector geometries, the background increases less with increasing sample thickness for the former.

\subsection{Signal-to-noise ratio}

Based on the calculated spectra, we calculated the $\mathrm{S} / \mathrm{N}$ and $\mathrm{P} / \mathrm{B}$ with respect to the $K \alpha$ peaks of $\mathrm{Zn}$ and As for the "bio" and "chip" samples respectively. The signal and the background are integrated within the FWHM of the XRF peak of the feature element. Figure 11 shows the S/N and P/B as a function of collection semi-angle $\phi$, for the modeled "bio" sample with $100 \mathrm{~nm}$ water layers, and for the three different detector geometries. (Similar trends were found for the other "bio" and "chip" samples considered above; see Supplementary). We found that the forward-scattering geometry is always the worst in terms of S/N or $\mathrm{P} / \mathrm{B}$, because of the maximized scattering at $0^{\circ}$ (due to the scattering cross sections favoring the forward direction) [53, p. 209, 220], and the possibly large self-absorption effect in the transmission geometry. It is also not difficult to understand that in both the forward- and back-scattering geometries, the S/N always increases with increasing collection semi-angle of the detector. This is because the scattering background decreases as one gets closer to $90^{\circ}$ to the incident beam. In the case of a detector at $90^{\circ}$, the $\mathrm{P} / \mathrm{B}$ goes down with increasing $\phi$ for the same reason, which is in consistent with the theoretical results by Hanson[58], where it was shown that the scattering background in the $90^{\circ}$ geometry should increase significantly faster than the increase of the total solid angle when the collection semi-angle is above $\sim 2^{\circ}$, for light matrix elements and incident energy below $20 \mathrm{KeV}$. In spite of that, however, the $\mathrm{S} / \mathrm{N}$ still increases with $\phi$ in the $90^{\circ}$ geometry. In other words, even at $90^{\circ}$, a larger detector is better. We did see slight decreases in $\mathrm{S} / \mathrm{N}$ at $\phi>45^{\circ}$ for 
the "bio" samples with $2 \times 10 \mu \mathrm{m}(\sim 1 \%$ decrease $)$ and $2 \times 100 \mu \mathrm{m}(\sim 9 \%$ decrease $)$ water layers. This is because when $\phi>45^{\circ}$, part of the detector is collecting photons emergent from the backside of the sample, thus resulting in a transmission geometry and increased self-absorption effect. One might argue that the increase in scattering background when the collection angle is increased can also cause a decrease in $\mathrm{S} / \mathrm{N}$. However, we will show in Section 4.3 that, in most cases, the $\mathrm{S} / \mathrm{N}$ will increase with increasing detector collection semi-angle, when self-absorption effect is excluded and the background is solely from Rayleigh and Compton scattering by the sample.

With calculations of the $\mathrm{S} / \mathrm{N}$ as a function of $\phi$ for different sample and detector geometries at hand, we can use these data to compare the performance of different detector setups. Figure 12 shows the collection semi-angle needed for a detector at $180^{\circ}$ in order to give the same $\mathrm{S} / \mathrm{N}$ as a detector centered at $0^{\circ}$, for both the "bio" sample with varying thickness water layers, and the "chip" sample with varying thickness Si layers. Figure 13 shows the same comparison between detectors at $90^{\circ}$ and $180^{\circ}$. In light of the elastic or Rayleigh scattering minimum, it is not surprising to find that a detector at $90^{\circ}$ always needs the smallest collection semi-angle to obtain the same $\mathrm{S} / \mathrm{N}$. For detectors at $0^{\circ}$ and $180^{\circ}$, the $\mathrm{S} / \mathrm{N}$ versus $\phi$ relations are almost the same when the sample is thin (as for $0.5 \mathrm{~nm}$ of As on top of $0.5 \mu \mathrm{m}$ of Si in the thin "chip" sample); however, when the sample gets thicker, scattering background and absorption artifacts become non-trivial and affect the $0^{\circ}$ geometry more (due to its transmission geometry), so that $\mathrm{S} / \mathrm{N}$ considerations favor the $180^{\circ}$ geometry.

\subsection{Comparison between typical Vortex detector $\left(90^{\circ}\right)$ and Maia detector $\left(180^{\circ}\right)$ geometries}

Figures 12 and 13 tells us that if we have a detector subtending a certain solid angle, it is always best to put it at $90^{\circ}$ in order to obtain the highest $\mathrm{S} / \mathrm{N}$; however, how does a larger detector at $180^{\circ}$ compare with a small detector at $90^{\circ}$ ? Let us consider the case of the 384-element Maia [31] detector centered at $180^{\circ}$ versus a single element Vortex EM (Hitachi High-Technologies Science America, Inc.) detector at $90^{\circ}$. For the Maia detector, we use the same semi-angle range of $\phi=13.9-52.6^{\circ}$ (approximating the Maia detector with an annular sensitive area as employed in a recent study [32]). For the Vortex EM detector, we assume an active area of $50 \mathrm{~mm}^{2}$ at a distance of $30 \mathrm{~mm}$ from the sample, giving a semi-angle $\phi=6.7^{\circ}$; we also assume that the sample is positioned at $45^{\circ}$ to the incident beam (parameters that match the experimental test of Section 3.5). Figures 14 and 15 show the minimum number of incident photons required according to the $S / N \geq 3$ rule, for the Vortex EM and Maia detectors respectively. From these plots we can see that, for the "chip" sample and the "bio" sample with thin water layers, the Maia detector requires a smaller number of illuminating photons to get the same S/N. However, as the water thickness increases, the Vortex EM detector starts to outperform the Maia detector. With thinner samples, the scattering background is small, so the intensity of the signal is the dominant restricting factor in $\mathrm{S} / \mathrm{N}$; when the sample gets thicker, there is increased scattering from the sample, so that reducing the background will be more important in 
increasing the $\mathrm{S} / \mathrm{N}$.

\subsection{The effect of scattering on $S / N$}

Assume an ideal sample with no self-absorption. Since fluorescence emission is isotropic, the intensity of the feature signal $\left(\bar{n} I_{f}=\bar{n} I_{p}-\bar{n} I_{a}\right)$ is linearly proportional to the collection solid angle $\Omega$ of the detector, or

$$
\bar{n} I_{f}=\bar{n} C\left(\frac{\mu_{\mathrm{YX}}}{\rho}\right) \frac{\Omega}{4 \pi}=\bar{n} C a \Omega,
$$

where $\mu_{\mathrm{YX}} / \rho$ is the mass coefficient for the production of the XRF photons of interest, and $C$ is the (mass) concentration of the feature element. If we assume that the background comes only from scattering by the sample, and the intensity of the background is a fraction of the total Compton and Rayleigh scattering signal, then the intensity of the background will be

$$
\bar{n} I_{a}=\bar{n} f \int \frac{d\left(\mu_{\text {scatt }} / \rho\right)}{d \Omega} d \Omega=\bar{n} f g(\Omega),
$$

where $\mu_{\text {scatt }} / \rho$ is the mass coefficient for the total (Rayleigh and Compton) scattering from the sample matrix, and $f$ is the fraction of the scattered photons that constitute the background at the XRF peak (as a result of the tailing of the scattering peaks due to incomplete charge collection). Therefore, the $\mathrm{S} / \mathrm{N}$ can be expressed as

$$
\begin{aligned}
\mathrm{S} / \mathrm{N} & =\sqrt{\bar{n}} \frac{I_{f}}{\sqrt{I_{f}+2 I_{a}}} \\
& =\sqrt{\bar{n}} \frac{C a \Omega}{\sqrt{C a \Omega+2 f g(\Omega)}}
\end{aligned}
$$

In Figure 16, we plot the contrast ratio $\Theta$ as a function of $C$ and $\phi$ for a detector at $90^{\circ}$ for a "bio"-like sample, which in this case consists of a "bio"-like sample with infinitesimal thickness (that is, no self-absorption) and a composition of $15 \mathrm{wt} \%$ of protein, $85 \mathrm{wt} \%$ of water, and a varied trace concentration $C$ of $\mathrm{Zn}$. We find that, in general, the $\mathrm{S} / \mathrm{N}$ always increases with increasing collection semi-angle $\phi$. The increase in $\mathrm{S} / \mathrm{N}$ is more remarkable when the concentration of $\mathrm{Zn}$ is higher. For samples with very low trace amount of $\mathrm{Zn}$, increasing $\phi$ after a certain angle will not give noticeable boosts in $\mathrm{S} / \mathrm{N}$ (increasing $\phi$ from $10^{\circ}$ to $60^{\circ}$ only gives an increase in $\mathrm{S} / \mathrm{N}$ of less than $2 \%$ when $C=10^{-9}$ ), due to a disproportionate increase in scattering relative to fluorescence signals. We did the same calculation for an ideal "chip" sample and obtained similar scalings, and indeed one would expect similar trends to apply to other sample types.

\section{Conclusions}

Based on the Sherman equation of the first order, we have developed an analytical model for simulating the X-ray fluorescence (XRF) spectrum of 2D-extended layered samples including background signals from 
elastic and Compton scattering, which gives results in good agreement with the experiment. This model was applied to two test samples, one simulating a biological cell and another simulating a doped silicon chip. We found that the forward-scattering geometry is always the worst in terms of $\mathrm{S} / \mathrm{N}$, while detectors at $90^{\circ}$ always give the highest $\mathrm{S} / \mathrm{N}$. In all of the three detector geometries, a larger collection angle leads to a larger $\mathrm{S} / \mathrm{N}$, and the increase in $\mathrm{S} / \mathrm{N}$ is more remarkable when the concentration of the feature element is higher. For detecting very low trace amount of elements in a thick sample with a detector at $90^{\circ}$, increasing the detector collection semi-angle $\phi$ beyond $10^{\circ}$ will not give any noticeable boosts in $\mathrm{S} / \mathrm{N}$. Compared to a large detector at $180^{\circ}$, a small detector at $90^{\circ}$ is less sensitive to the change of sample thickness. Because the scattering background is minimized at $90^{\circ}$, the increase (or decrease) in the scattering background due to the change in sample thickness does not affect the $\mathrm{S} / \mathrm{N}$ as much as a detector at $180^{\circ}$. Therefore, when detecting trace amount of element in a thick sample, it is better to use a small detector at $90^{\circ}$; however, when the feature element is high in concentration or when the sample is very thin, using a large detector at $180^{\circ}$ will give a higher $\mathrm{S} / \mathrm{N}$.

Plural scattering and mutual enhancement of fluorescence are not included in our calculations. More accurate results may be achieved by including these higher order terms in the Sherman equation, and also by including the Compton broadening effect. In addition, all samples are considered in vacuum (except for the comparison with the experiment). In real cases with gas environment, attenuation by the gas should be added, and hence the distance between the sample and the detector will modify the conclusions drawn above.

\section{Acknowledgments}

This work was supported by the US Department of Energy, Office of Science, Basic Energy Sciences program under contract DE-AC02-06CH11357, and by the National Institutes of Health under grant R01 GM104530. We would also like to thank Mark Rivers for his constructive suggestions.

\section{References}

[1] T. Paunesku, S. Vogt, J. Maser, B. Lai, G. Woloschak, X-ray fluorescence microprobe imaging in biology and medicine, Journal of Cellular Biochemistry 99 (2006) 1489-1502.

[2] C. Fahrni, Biological applications of x-ray fluorescence microscopy: exploring the subcellular topography and speciation of transition metals, Current Opinion in Chemical Biology 11 (2007) 121-127.

[3] M. Szczerbowska-Boruchowska, X-ray fluorescence spectrometry, an analytical tool in neurochemical research, X-ray Spectrometry 37 (1) (2008) 21-31.

[4] M. D. de Jonge, S. Vogt, Hard x-ray fluorescence tomography - an emerging tool for structural visualization, Current Opinion in Structural Biology 20 (5) (2010) 606-614.

[5] Z. Qin, J. A. Caruso, B. Lai, A. Matusch, J. S. Becker, Trace metal imaging with high spatial resolution: applications in biomedicine, Metallomics 3 (1) (2011) 28-37. 
[6] U. E. A. Fittschen, G. Falkenberg, Trends in environmental science using microscopic x-ray fluorescence, Spectrochimica Acta B 66 (8) (2011) 567-580.

[7] S. Majumdar, J. R. Peralta-Videa, H. Castillo-Michel, J. Hong, C. M. Rico, J. L. Gardea-Torresdey, Applications of synchrotron $\mu$-xrf to study the distribution of biologically important elements in different environmental matrices: A review, Analytica chimica acta 755 (2012) 1-16.

[8] M. J. Pushie, I. J. Pickering, M. Korbas, M. J. Hackett, G. N. George, Elemental and chemically specific x-ray fluorescence imaging of biological systems, Chemical Reviews 114 (2014) 140807162425009.

[9] T. Buonassisi, A. A. Istratov, M. A. Marcus, B. Lai, Z. Cai, S. M. Heald, E. R. Weber, Engineering metal-impurity nanodefects for low-cost solar cells, Nature Materials 4 (9) (2005) 676-679.

[10] M. Bertoni, D. Fenning, M. Rinio, V. Rose, M. Holt, J. Maser, T. Buonassisi, Nanoprobe x-ray fluorescence characterization of defects in large-area solar cells, Energy \& Environmental Science 4 (10) (2011) 4252-4257.

[11] S. A. Johansson, PIXE: a novel technique for elemental analysis, Endeavour 13 (2) (1989) 48-53.

[12] C. G. Ryan, Quantitative trace element imaging using PIXE and the nuclear microprobe, International Journal of Imaging Systems and Technology 11 (4) (2000) 219-230.

[13] R. Castaing, Electron probe microanalysis, Advances in electronics and electron physics 13 (1960) 317-386.

[14] A. LeFurgey, P. Ingram, Calcium measurements with electron probe x-ray and electron energy loss analysis, Environmental Health Perspectives 84 (1990) 57-73.

[15] J. Kirz, D. Sayre, J. Dilger, Comparative analysis of x-ray emission microscopies for biological specimens, in: D. F. Parsons (Ed.), Short Wavelength Microscopy, Vol. 306 of Annals of the New York Academy of Sciences, New York, 1978, pp. $291-305$.

[16] J. Kirz, Mapping the distribution of particular atomic species, in: D. F. Parsons (Ed.), Ultrasoft X-ray Microscopy: Its Application to Biological and Physical Sciences, Vol. 342 of Annals of the New York Academy of Sciences, New York, 1980, pp. 273-287.

[17] J. Kirz, Specimen damage considerations in biological microprobe analysis, in: Scanning Electron Microscopy, Vol. 2, SEM Inc., Chicago, 1980, pp. 239-249.

[18] K. Jones, B. Gordon, A. Hanson, W. M. Kwiatek, J. Pounds, X-ray fluorescence with synchrotron radiation, Ultramicroscopy 24 (2) (1988) 313-328.

[19] C. J. Sparks, Jr., X-ray fluorescence microprobe for chemical analysis, in: H. Winick, S. Doniach (Eds.), Synchrotron Radiation Research, Plenum Press, New York, 1980, pp. 459-512.

[20] S. Agostinelli, J. Allison, K. a. Amako, J. Apostolakis, H. Araujo, P. Arce, M. Asai, D. Axen, S. Banerjee, G. Barrand, et al., Geant4a simulation toolkit, Nuclear instruments and methods in physics research section A: Accelerators, Spectrometers, Detectors and Associated Equipment 506 (3) (2003) 250-303.

[21] J. Brown, M. R. Dimmock, J. E. Gillam, D. M. Paganin, MULECS: The Monash University low energy compton scattering package, in: D. Townsend (Ed.), 2011 IEEE Nuclear Science Sympoisum and Medical Imaging Conference, IEEE, 2011, pp. 1385-1389. doi:10.1109/NSSMIC.2011.6154623.

[22] J. M. C. Brown, M. R. Dimmock, J. E. Gillam, D. M. Paganin, A low energy bound atomic electron compton scattering model for Geant4, Nuclear Instruments and Methods in Physics Research B 338 (2014) $77-88$. doi:10.1016/j.nimb.2014.07.042.

[23] L. Vincze, K. Janssen, F. Adams, A general Monte Carlo simulation of energy-dispersive x-ray fluorescence spectrometersI: Unpolarized radiation, homogeneous samples, Spectrochimica Acta B 48 (4) (1993) 553-573.

[24] L. Vincze, K. Janssens, F. Adams, M. L. Rivers, K. W. Jones, A general Monte Carlo simulation of ED-XRF spectrometers. II: Polarized monochromatic radiation, homogeneous samples, Spectrochimica Acta B 50 (2) (1995) $127-147$.

[25] L. Vincze, K. Janssens, F. Adams, K. W. Jones, A general Monte Carlo simulation of energy-dispersive x-ray fluorescence 
spectrometers. Part 3. Polarized polychromatic radiation, homogeneous samples, Spectrochimica Acta B 50 (12) (1995) $1481-1500$.

[26] L. Vincze, K. Janssens, B. Vekemans, F. Adams, Monte Carlo simulation of x-ray fluorescence spectra: Part 4. Photon scattering at high x-ray energies, Spectrochimica Acta B 54 (12) (1999) 1711-1722.

[27] T. Schoonjans, L. Vincze, V. A. Solé, M. Sanchez del Rio, P. Brondeel, G. Silversmit, K. Appel, C. Ferrero, A general Monte Carlo simulation of energy dispersive x-ray fluorescence spectrometers - part 5. polarized radiation, stratified samples, cascade effects, M-lines, Spectrochimica Acta B 70 (2012) 10-23.

[32] C. G. Ryan, D. P. Siddons, R. Kirkham, Z. Y. Li, M. D. de Jonge, D. J. Paterson, A. Kuczewski, D. L. Howard, P. A. Dunn, G. Falkenberg, U. Boesenberg, G. De Geronimo, L. A. Fisher, A. Halfpenny, M. J. Lintern, E. Lombi, K. A. Dyl, M. Jensen, G. F. Moorhead, J. S. Cleverley, R. M. Hough, B. Godel, S. J. Barnes, S. A. James, K. M. Spiers, M. Alfeld, G. Wellenreuther, Z. Vukmanovic, S. Borg, Maia x-ray fluorescence imaging: Capturing detail in complex natural samples, in: G. Falkenberger, C. G. Schroer (Eds.), $22^{\text {nd }}$ International Congress on X-ray Optics and Microanalysis, Vol. 499, 2014, p. 01200.

[33] A. M. Kim, S. Vogt, T. V. O'Halloran, T. K. Woodruff, Zinc availability regulates exit from meiosis in maturing mammalian oocytes, Nature Chemical Biology 6 (9) (2010) 674-681. doi:10.1038/NCHEMBIO.419.

[34] R. Kirkham, P. A. Dunn, A. J. Kuczewski, D. P. Siddons, R. Dodanwela, G. F. Moorhead, C. G. Ryan, G. De Geronimo, R. Beuttenmuller, D. Pinelli, M. Pfeffer, P. Davey, M. Jensen, D. J. Paterson, M. D. de Jonge, D. L. Howard, M. Küsel, J. McKinlay, R. Garrett, I. Gentle, K. Nugent, S. Wilkins, The Maia spectroscopy detector system: Engineering for integrated pulse capture, low-latency scanning and real-time processing, in: R. Garrett, I. Gentle, K. Nugent, S. Wilkins (Eds.), The $10^{\text {th }}$ International Conference on Synchrotron Radiation Instrumentation, Vol. 1234, American Institute of Physics Conference Proceedings, 2010, pp. 240-243.

[35] O. Klein, Y. Nishina, The scattering of light by free electrons according to Dirac's new relativistic dynamics, Nature 122 (1928) 398-399.

[36] O. Klein, Y. Nishina, Über die Streuung von Strahlung durch freie Elektronen nach der neuen relativischen Quantendynamik von Dirac, Zeitschrift für Physik 52 (11-12) (1929) 853-868. doi:10.1007/BF01366453.

[37] J. Hubbell, W. J. Veigele, E. Briggs, R. Brown, D. Cromer, R. Howerton, Atomic form factors, incoherent scattering functions, and photon scattering cross sections, Journal of Physical and Chemical Reference Data 4 (3) (1975) $471-538$.

[38] P. Auger, Sur l'effet photoélectrique composé, Journal de Physique et Le Radium 6 (6) (1925) 205-208. doi:10.1051/jphysrad:0192500606020500.

[39] M. O. Krause, Atomic radiative and radiationless yields for $K$ and $L$ shells, Journal of Physical and Chemical Reference Data 8 (1979) 307-327. 
aspects of electron microscopy and microbeam analysis, Wiley, New York, 1975, pp. 205-227.

[41] D. Sayre, J. Kirz, R. Feder, D. M. Kim, E. Spiller, Transmission microscopy of unmodified biological materials: Comparative radiation dosages with electrons and ultrasoft x-ray photons, Ultramicroscopy 2 (1977) 337-341.

[42] D. Sayre, J. Kirz, R. Feder, D. M. Kim, E. Spiller, Potential operating region for ultrasoft x-ray microscopy of biological specimens, Science 196 (1977) 1339-1340.

[43] R. Jenkins, Quantitative X-ray spectrometry, CRC Press, 1995.

[44] R. Van Grieken, A. Markowicz, Handbook of X-ray Spectrometry, CRC Press, 2001.

[45] E. Clayton, P. Duerden, D. D. Cohen, A discussion of PIXAN and PIXANPC: The AAEC PIXE analysis computer packages, Nuclear Instruments and Methods in Physics Research Section B: Beam Interactions with Materials and Atoms 22 (1) (1987) 64-67.

[46] C. Ryan, E. Clayton, W. Griffin, S. Sie, D. Cousens, SNIP, a statistics-sensitive background treatment for the quantitative analysis of PIXE spectra in geoscience applications, Nuclear Instruments and Methods in Physics Research B 34 (3) (1988) 396-402.

[47] A. Rose, Unified approach to performance of photographic film, television pickup tubes, and human eye, Journal of the Society of Motion Picture Engineers 47 (1946) 273-294.

[48] J. Brink, S. Ludtke, C.-Y. Yang, Z.-W. Gu, S. Wakil, W. Chiu, Quaternary structure of human fatty acid synthase by electron cryomicroscopy, Proceedings of the National Academy of Sciences of the USA 99 (1) (2002) 138-143. doi:10.1073/pnas.012589499.

[49] R. Cesareo, G. E. Gigante, A. L. Hanson, A method for forward energy-dispersive x-ray fluorescence analysis of thin and intermediate samples, Nuclear Instruments and Methods in Physics Research Section B: Beam Interactions with Materials and Atoms 145 (3) (1998) 434-448.

[50] B. Golosio, T. Schoonjans, A. Brunetti, P. Oliva, G. L. Masala, Monte Carlo simulation of x-ray imaging and spectroscopy experiments using quadric geometry and variance reduction techniques, Computer Physics Communications 185 (3) (2014) 1044-1052.

[51] Y. Sun, Sim-xrf source code, https://github.com/gromitsun/sim-xrf/, [Online] (2014).

[52] J. Als-Nielsen, D. McMorrow, Elements of modern X-ray physics, John Wiley \& Sons, 2011.

[53] N. A. Dyson, N. A. Dyson, X-rays in Atomic and Nuclear Physics, Cambridge University Press, 2005.

[54] T. Schoonjans, A. Brunetti, B. Golosio, M. S. del Rio, V. A. Solé, C. Ferrero, L. Vincze, The xraylib library for x-raymatter interactions. Recent developments, Spectrochimica Acta B 66 (11-12) (2011) 776-784.

[55] L. Kissel, RTAB: the Rayleigh scattering database, Radiation Physics and Chemistry 59 (2) (2000) 185-200.

[56] J. Sherman, The theoretical derivation of fluorescent x-ray intensities from mixtures, Spectrochimica Acta 7 (1955) 283306.

[57] F. Tokanai, H. Sakurai, S. Gunji, S. Motegi, H. Toyokawa, M. Suzuki, K. Hirota, S. Kishimoto, K. Hayashida, Hard x-ray polarization measured with a Compton polarimeter at synchrotron radiation facility, Nuclear Instruments and Methods in Physics Research A 530 (3) (2004) 446-452.

[58] A. Hanson, A method for selecting detector apertures for use in \{SRIXE\}, Nuclear Instruments and Methods in Physics Research Section A: Accelerators, Spectrometers, Detectors and Associated Equipment 260 (1) (1987) $264-$ 275. doi:http://dx.doi.org/10.1016/0168-9002(87)90412-8.

URL http://www.sciencedirect.com/science/article/pii/0168900287904128 


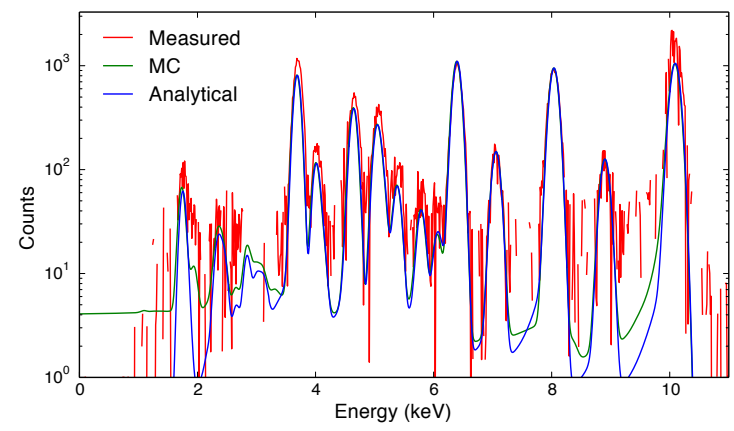

(a) Detector center at $50^{\circ}$.

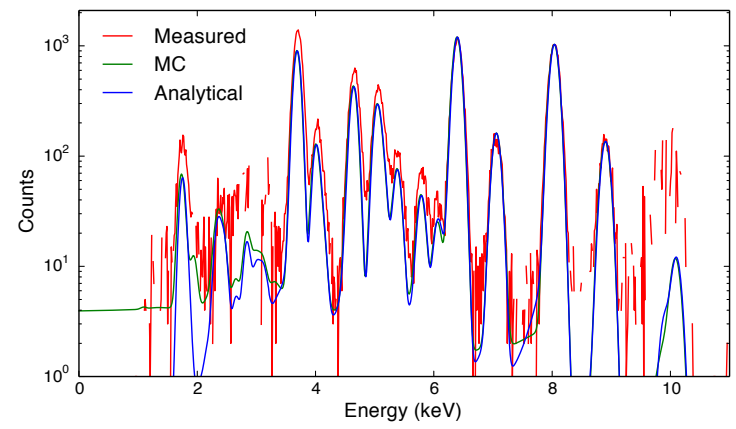

(b) Detector center at $90^{\circ}$.

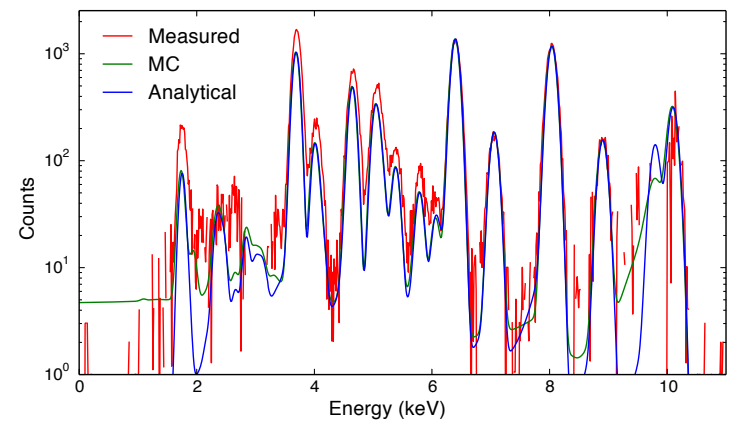

(c) Detector center at $124^{\circ}$.

Figure 7: Comparison between calculated and experimental spectra of the AXO standard as described in Section 3.5. Red lines are experimental data, blue lines are calculated spectra using analytical method, green lines are spectra from Monte Carlo simulations including Compton broadening and Si escape peaks. The calculated and simulated spectra were scaled with the experimental data using the Fe $K \alpha$ peak. Attenuation by the air was included. Note that our analytical method required $\sim 20$ seconds of computation in a single process on a laptop computer, while the XMI-MSIM Monte Carlo simulation required runs $\sim 10$ minutes to run on a four-core processor. 


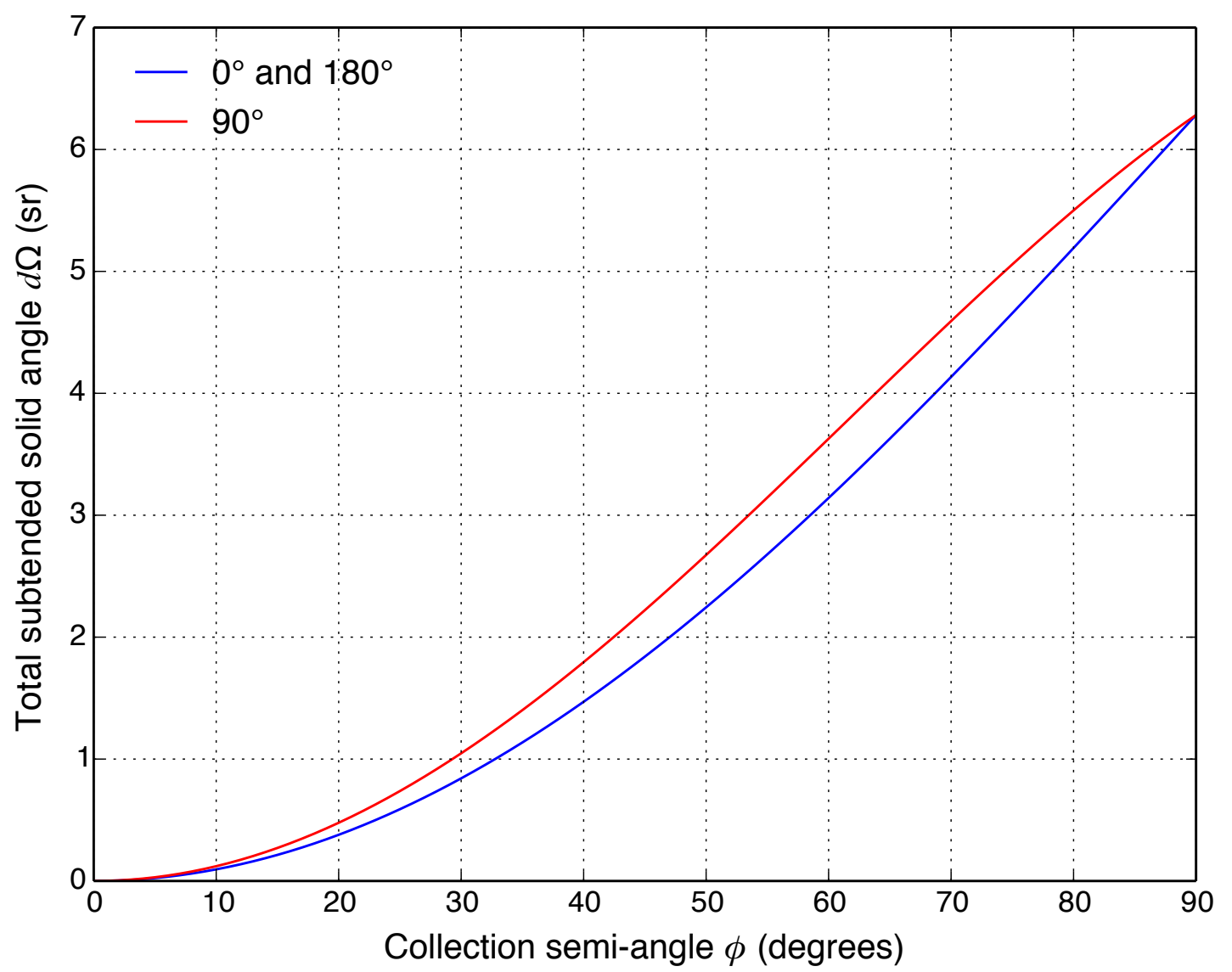

Figure 8: Plot of the solid angles subtended by detectors centered at $90^{\circ}$ versus $0^{\circ}$ and $180^{\circ}$ as a function of the collection semi-angle $\phi$, using detector geometries described in Table 2 . 


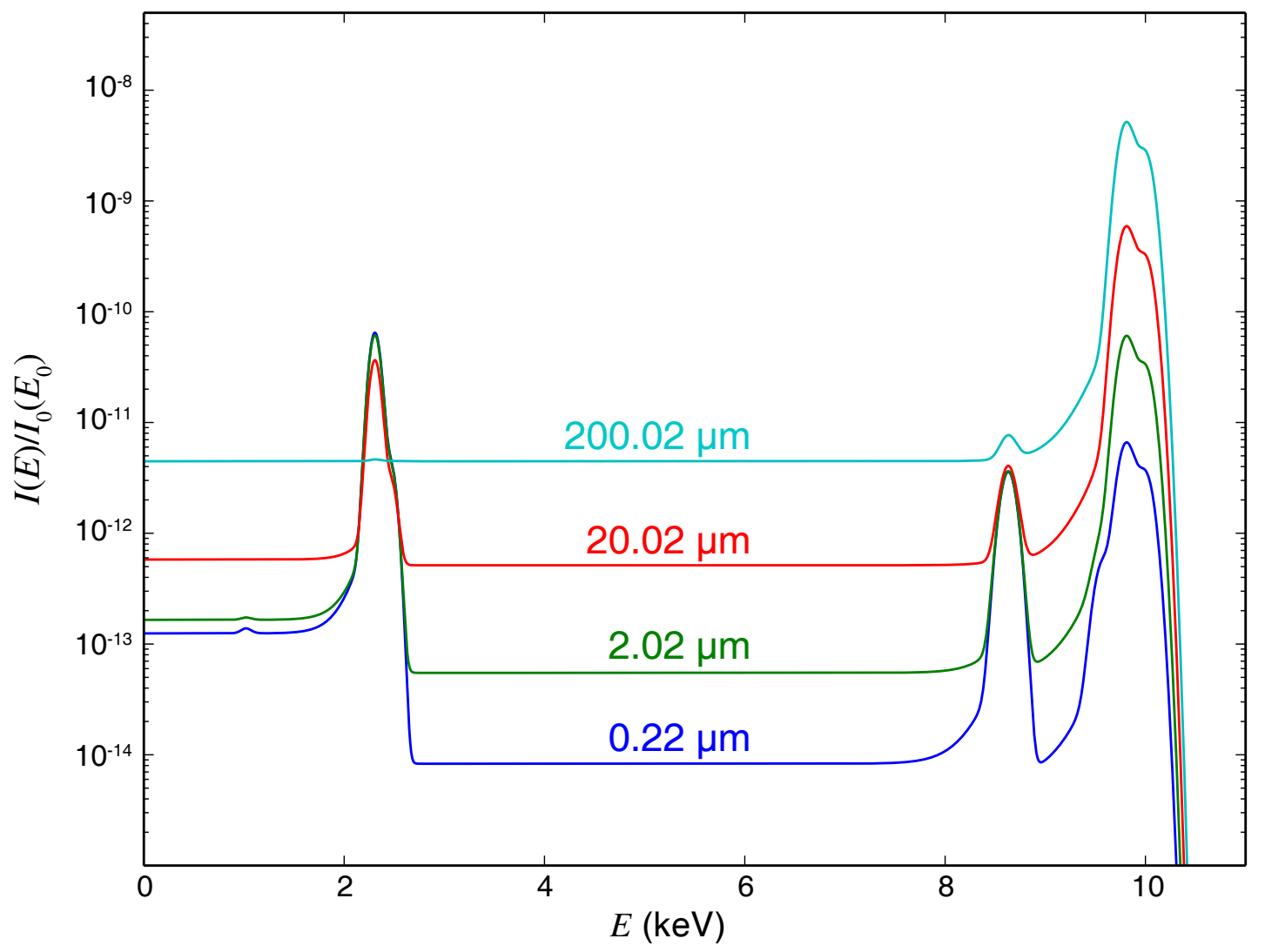

Figure 9: Calculated spectra of the modeled "bio" sample with varying water layer thickness $(2 \times 0.1 \mu \mathrm{m}, 2 \times 1 \mu \mathrm{m}, 2 \times 10 \mu \mathrm{m}$, and $2 \times 100 \mu \mathrm{m}$, total sample thicknesses labeled in legends). The incident energy is $E=10 \mathrm{keV}$. A detector at $90^{\circ}$ with collection semi-angle $\phi=5^{\circ}$ is assumed. The $\mathrm{S}$ peak at around $2 \mathrm{KeV}$ decreases with increased sample thickness due to increased absorption by the water layers, but the $\mathrm{Zn}$ peak with a much higher energy $(>8 \mathrm{keV})$ is affected much less. All spectra are normalized by the incident beam intensity $\left(I_{0}(E)\right)$, which also applies to Figure 10 . 


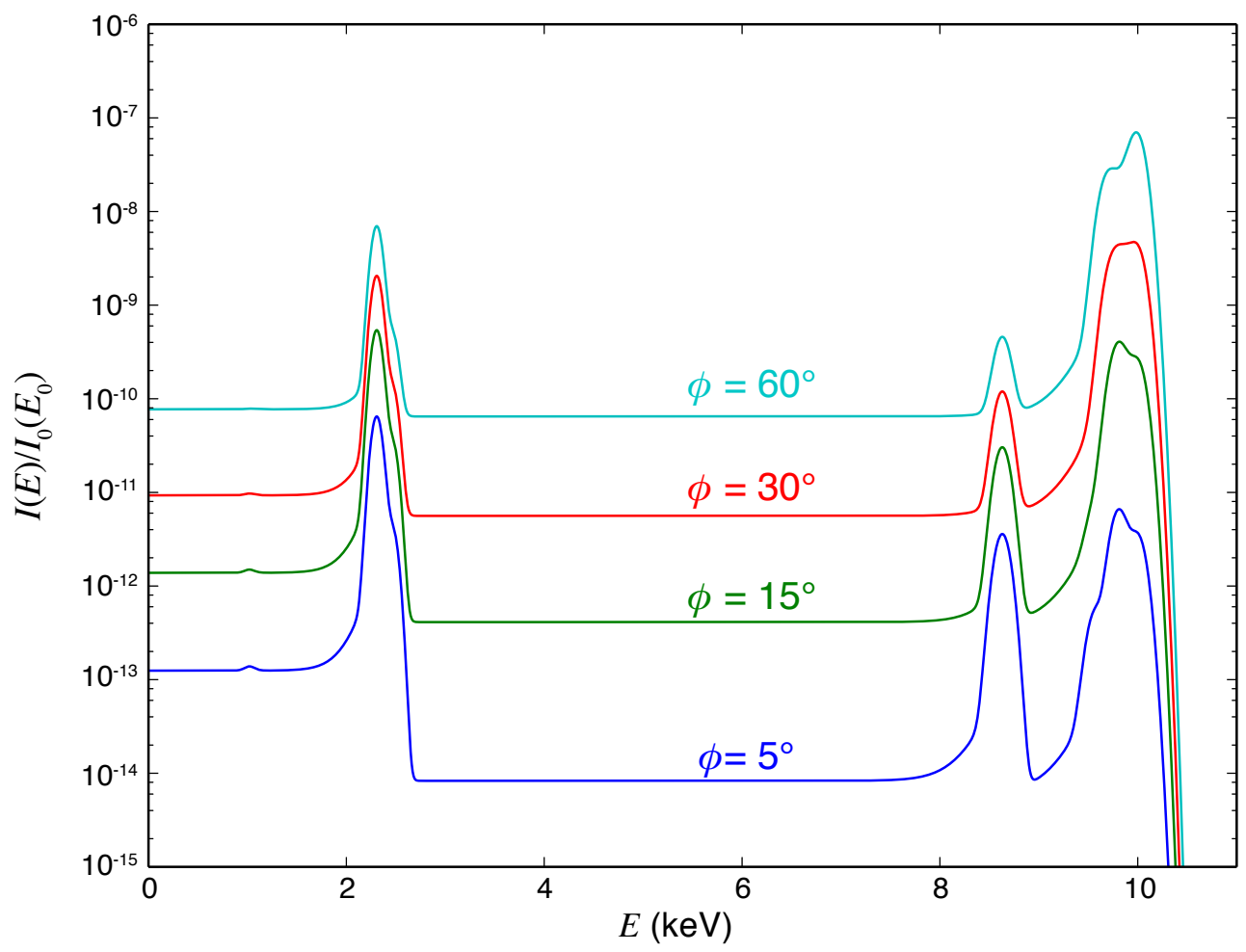

Figure 10: Calculated spectra of the modeled "bio" sample with $2 \times 100 \mathrm{~nm}$ water layers. The incident energy is $E=10 \mathrm{KeV}$. A detector at $90^{\circ}$ with varying collection semi-angle $\phi$ is assumed (labeled in legends). Both the scattering background and the XRF peaks increase as $\phi$ increases. While at larger $\phi$ the $\mathrm{P} / \mathrm{B}$ is smaller and $\mathrm{S} / \mathrm{N}$ is comparable with when $\phi$ is small, one needs fewer incident photons to detect the feature signal (i.e. to detect at least a certain number of photons from the feature signal) for a larger $\phi$. 


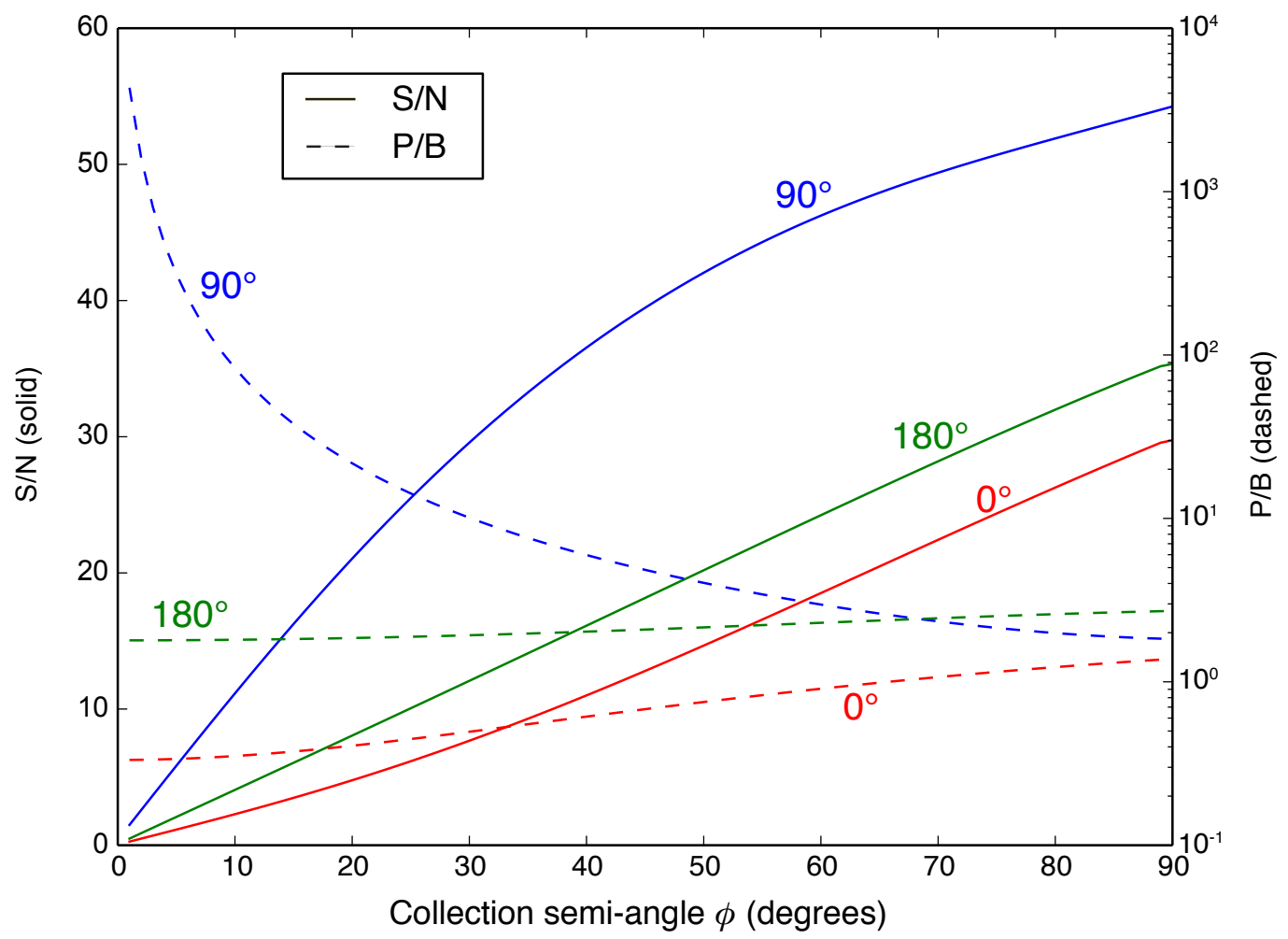

Figure 11: Signal to noise (S/N) and peak to background (P/B) ratios as a function of collection semi-angle $\phi$, for the modeled "bio" sample with $100 \mathrm{~nm}$ water layers. The $\mathrm{Zn} K \alpha$ peak is used for analysis. The incident energy is $E=10 \mathrm{KeV}$. Detectors at $0^{\circ}, 90^{\circ}$ and $180^{\circ}$ are considered. The signal and the background are integrated within the FWHM of the XRF peak. The calculation assumes a total of $10^{12}$ photons incident, or equivalently incident beam intensity $I_{0}(E)=10^{12}$ photons $/$ second with an acquisition time $t=1 \mathrm{~s}$. 


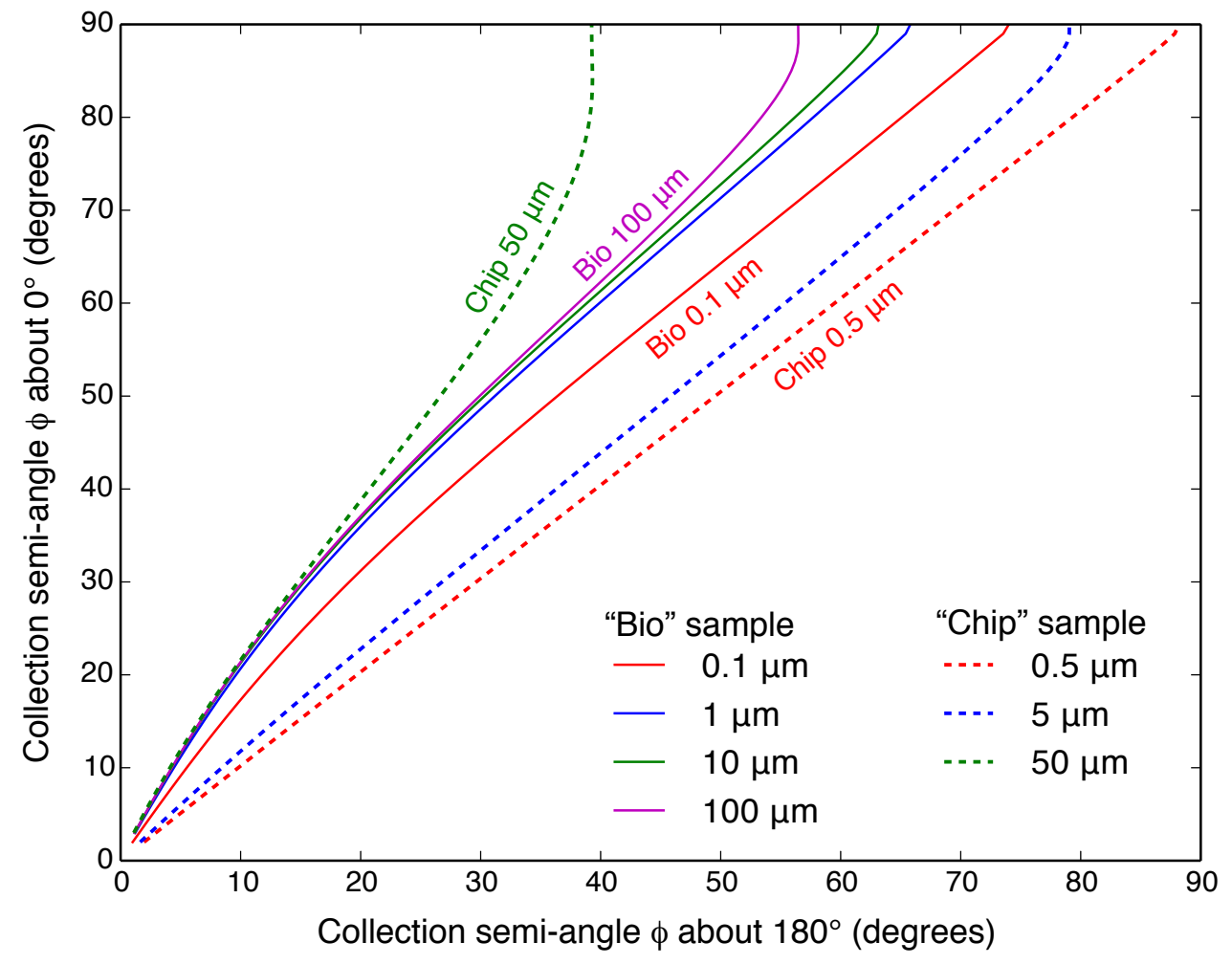

Figure 12: Collection semi-angle $\phi$ needed for a detector at $180^{\circ}$ in order to give the same $\mathrm{S} / \mathrm{N}$ as a detector centered at $0^{\circ}$, with the same number of photons incident. The calculations were done for the "bio" model sample with varying thickness water layers (solid lines), and the "chip" model sample with varying thickness Si layers (dashed lines). 


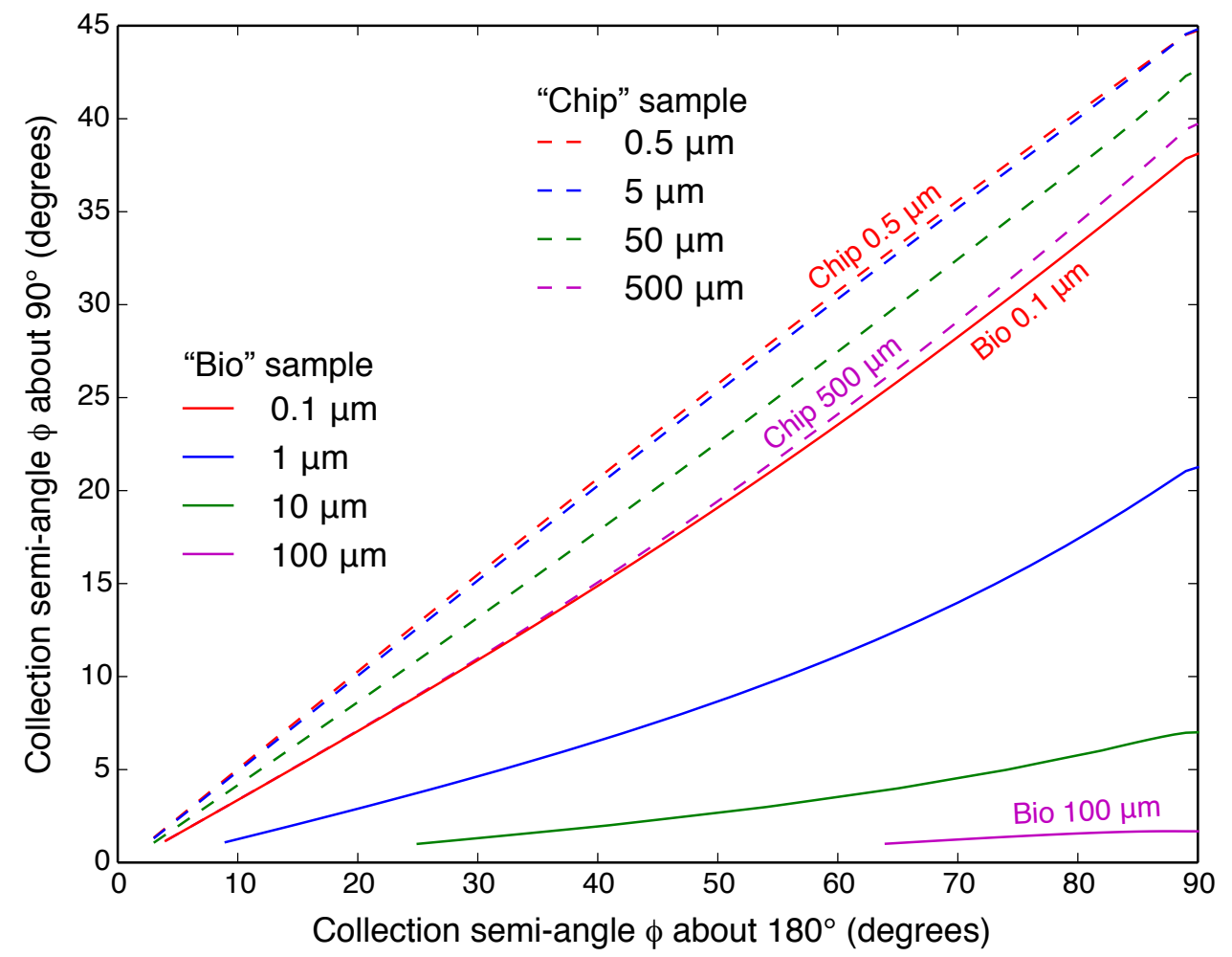

Figure 13: Collection semi-angle $\phi$ needed for a detector at $90^{\circ}$ in order to give the same $\mathrm{S} / \mathrm{N}$ as a detector centered at $180^{\circ}$, with the same number of photons incident. The calculations were done for the "bio" model sample with varying thickness water layers (solid lines), and the "chip" model sample with varying thickness Si layers (dashed lines). Note that the $Y$ scale is different from Figure 12 . 


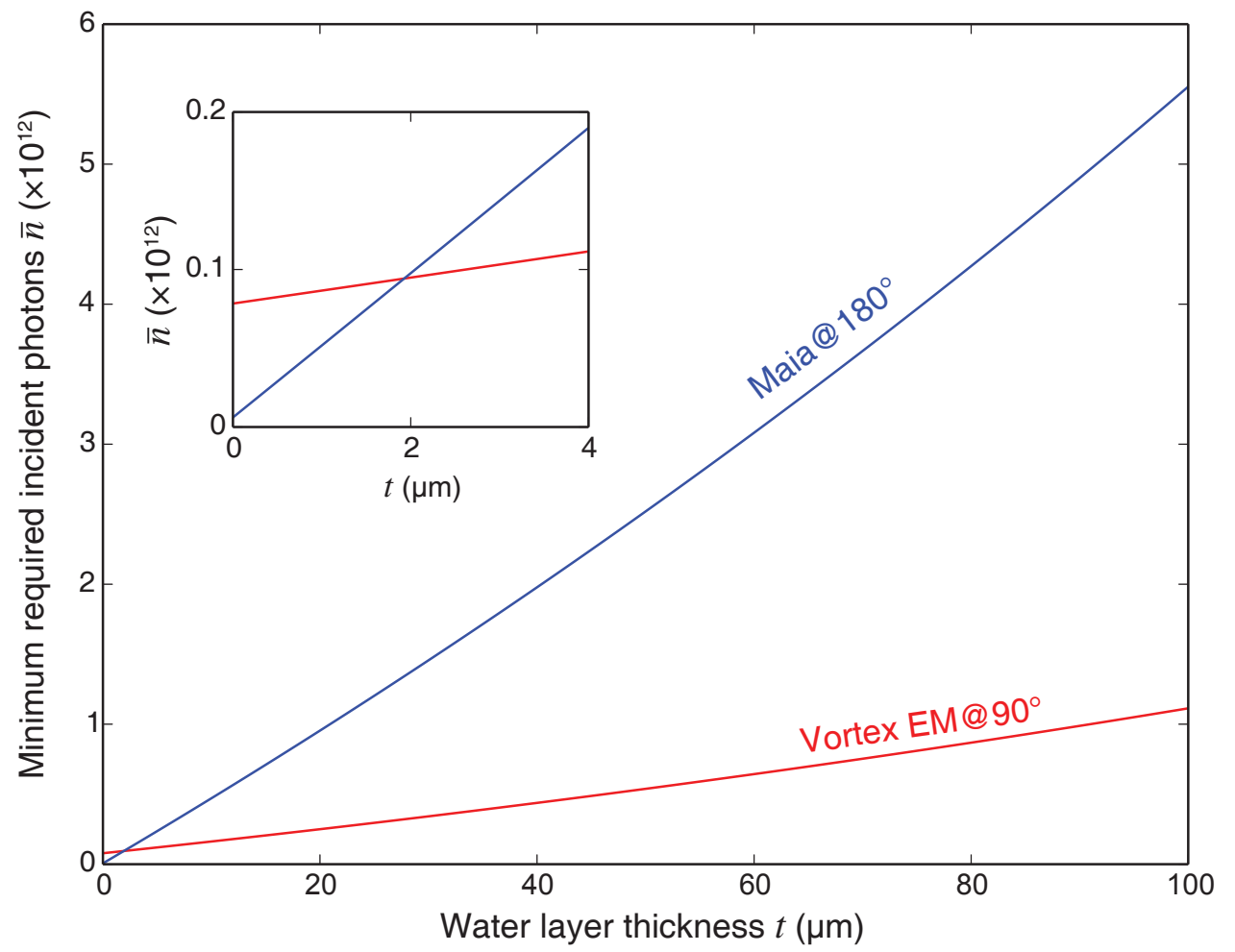

Figure 14: Minimum incident photons for $S / N \geq 3$ detection in the "bio" sample when the Vortex EM detector is used at $90^{\circ}$, and a Maia detector at $180^{\circ}$. 


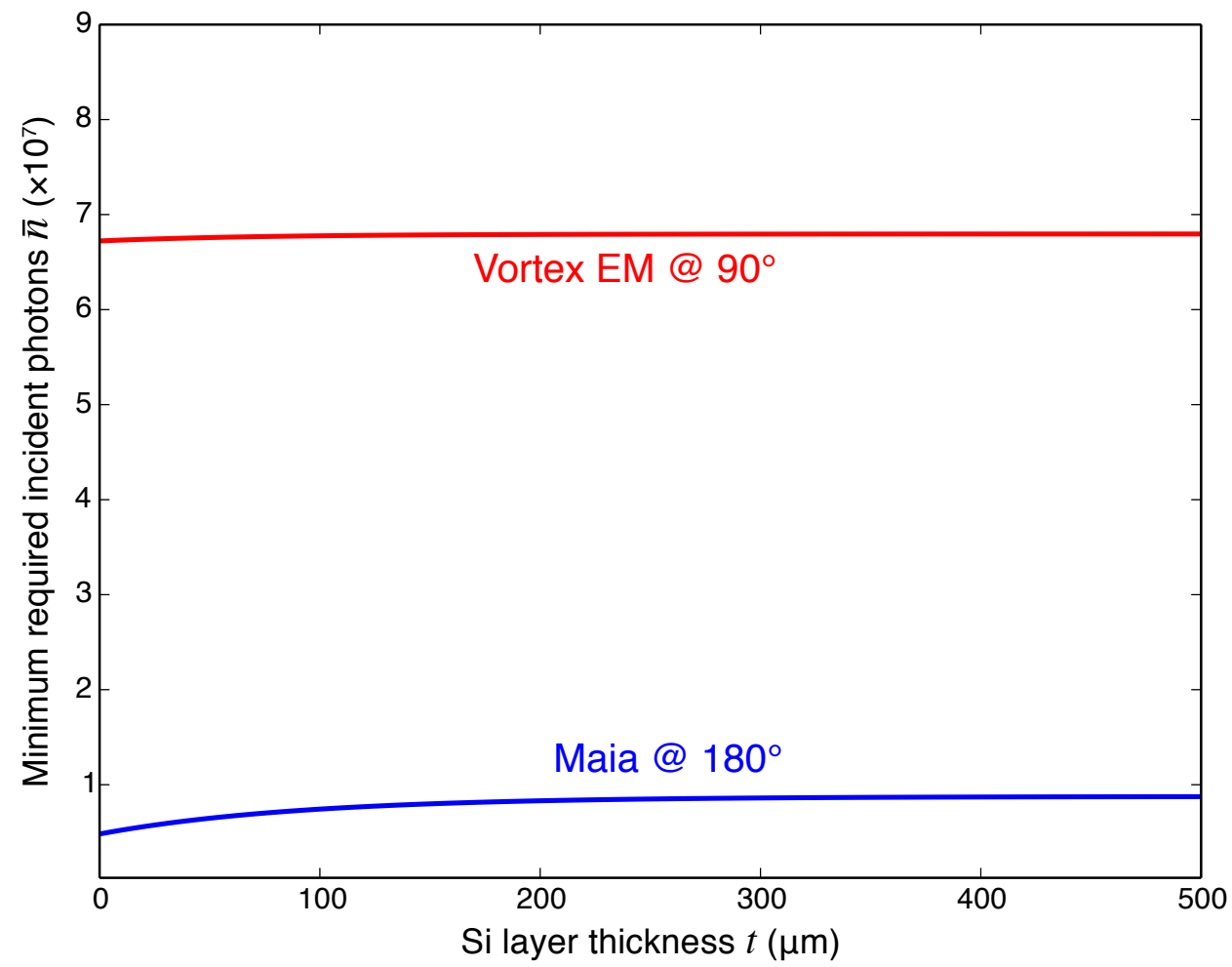

Figure 15: Minimum incident photons required by $S / N \geq 3$ detection in the "chip" sample when the Vortex EM detector is used at $90^{\circ}$, and a Maia detector at $180^{\circ}$. 

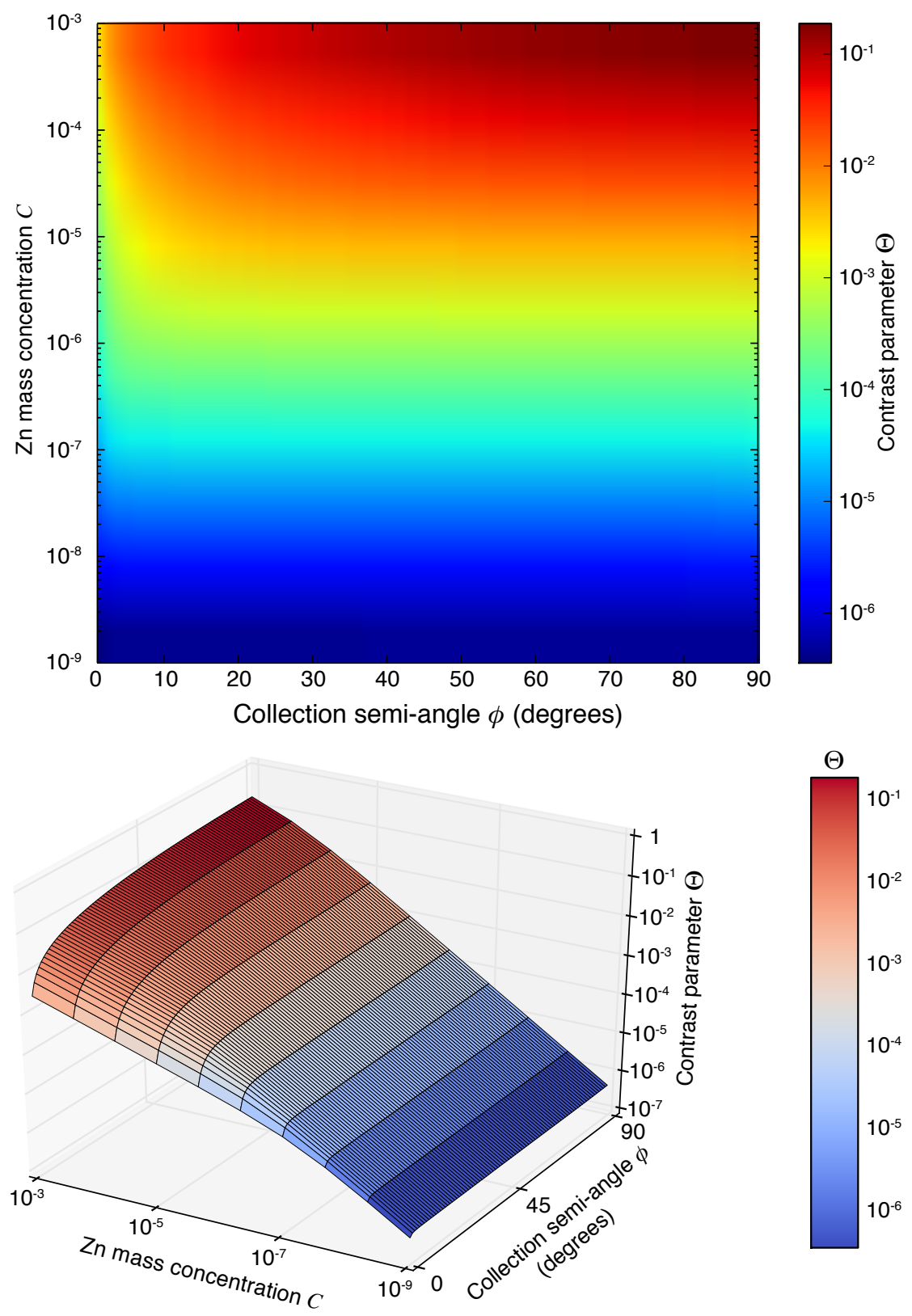

Figure 16: The contrast parameter $\Theta$ as a function of $\mathrm{Zn}$ concentration $C$ and $\phi$ of a detector at $90^{\circ}$, plotted both using an intensity scale and also using a 3D surface view. This calculation was done for an incident photon energy of $10 \mathrm{KeV}$ with a "bio"-like sample with infinitesimal thickness (so that self-absorption need not be accounted for) with 15 wt\% of protein, $85 \mathrm{wt} \%$ of water, and a trace amount $C$ (mass concentration) of $\mathrm{Zn}$. 